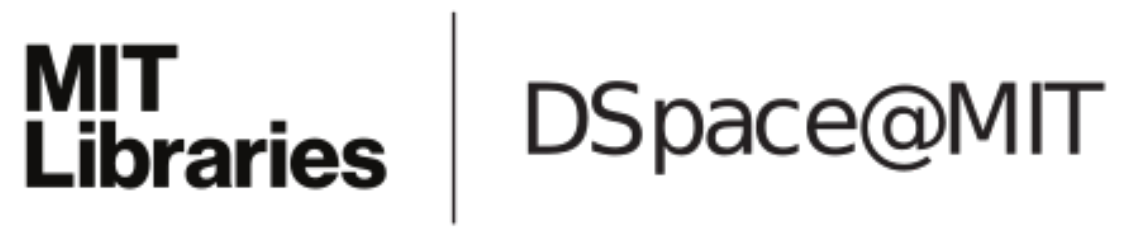

\author{
MIT Open Access Articles
}

THE CHEMICAL EVOLUTION OF THE
BOÖTES I ULTRA-FAINT DWARF GALAXY

The MIT Faculty has made this article openly available. Please share how this access benefits you. Your story matters.

Citation: Frebel, Anna, et al. "THE CHEMICAL EVOLUTION OF THE BOÖTES I ULTRA-FAINT DWARF GALAXY." The Astrophysical Journal 826, 2 (July 2016):110. (c) 2016 American Astronomical Society

As Published: http://dx.doi.org/10.3847/0004-637X/826/2/110

Publisher: IOP Publishing

Persistent URL: http://hdl.handle.net/1721.1/114026

Version: Final published version: final published article, as it appeared in a journal, conference proceedings, or other formally published context

Terms of Use: Article is made available in accordance with the publisher's policy and may be subject to US copyright law. Please refer to the publisher's site for terms of use. 


\title{
THE CHEMICAL EVOLUTION OF THE BOÖTES I ULTRA-FAINT DWARF GALAXY*
}

\author{
Anna Frebel ${ }^{1,2}$, John E. Norris ${ }^{3}$, Gerard Gilmore ${ }^{4}$, and Rosemary F. G. Wyse ${ }^{5}$ \\ ${ }^{1}$ Department of Physics and Kavli Institute for Astrophysics and Space Research, Massachusetts Institute of Technology, Cambridge, MA 02139, USA \\ ${ }_{2}^{2}$ Joint Institute for Nuclear Astrophysics-Center for Evolution of the Elements, East Lansing, MI 48824, USA \\ ${ }^{3}$ Research School of Astronomy and Astrophysics, Australian National University, Canberra, ACT, 2611, Australia \\ ${ }^{4}$ Institute of Astronomy, University of Cambridge, Cambridge CB3 OHA, UK \\ ${ }^{5}$ The Johns Hopkins University, Department of Physics \& Astronomy, Baltimore, MD 21218, USA \\ Received 2016 March 31; revised 2016 May 11; accepted 2016 May 11; published 2016 July 26
}

\begin{abstract}
We present chemical abundance measurements of two metal-poor red giant stars in the ultra-faint dwarf galaxy Boötes I, based on Magellan/MIKE high-resolution spectra. For Boo-980, with $[\mathrm{Fe} / \mathrm{H}]=-3.1$, we present the first elemental abundance measurements, while Boo-127, with $[\mathrm{Fe} / \mathrm{H}]=-2.0$, shows abundances in good agreement with previous measurements. Light and iron-peak element abundance ratios in the two Boötes I stars, as well as those of most other Boötes I members, collected from the literature, closely resemble those of regular metal-poor halo stars. Neutron-capture element abundances $\mathrm{Sr}$ and $\mathrm{Ba}$ are systematically lower than the main halo trend and also show a significant abundance spread. Overall, this is similar to what has been found for other ultrafaint dwarf galaxies. We apply corrections to the carbon abundances (commensurate with stellar evolutionary status) of the entire sample and find $21 \%$ of stars to be carbon-enhanced metal-poor (CEMP) stars, compared to $13 \%$ without using the carbon correction. We reassess the metallicity distribution functions for the CEMP stars and non-CEMP stars, and confirm earlier claims that CEMP stars might belong to a different, earlier population. Applying a set of abundance criteria to test to what extent Boötes I could be a surviving first galaxy suggests that it is one of the earliest assembled systems that perhaps received gas from accretion from other clouds in the system, or from swallowing a first galaxy or building block type object. This resulted in the two stellar populations observable today.
\end{abstract}

Key words: early universe - galaxies: dwarf - Galaxy: halo - Local Group - stars: abundances - stars: Population II

Supporting material: machine-readable table

\section{INTRODUCTION}

Dwarf satellite galaxies are versatile probes of chemical evolution, galactic halo assembly, and early galaxy formation processes. Ultra-faint dwarf galaxies $\left(L<10^{5} L_{\odot}\right)$ are particularly well-suited for testing these processes since they appear to have had limited star formation and chemical evolution, thus rendering them relatively simple and unevolved systems. What has made ultra-faint dwarfs particularly interesting is their overall low metallicity coupled with large metallicity spreads of several dex (e.g., Kirby et al. 2008). While stars with metallicities below $[\mathrm{Fe} / \mathrm{H}]=-3.0$ have been found in nearly all of these systems, the ultra-faint dwarfs completely lack higher-metallicity stars $[\mathrm{Fe} / \mathrm{H}]>-1.0$ showing that chemical enrichment did not proceed long enough to reach even close to the solar level.

Based on the chemical abundances of the seven brightest stars, the faintest of the ultra-faint dwarfs, Segue 1, was found to be a promising candidate for a surviving first galaxy (Frebel et al. 2014). Criteria for such a survivor include large spreads in $[\mathrm{Fe} / \mathrm{H}]$, halo-like $\alpha$-abundances at higher metallicity, and low neutron-capture element abundances, all of which are found among Segue 1 stars. Being the faintest of all ultra-faint dwarf galaxies, Segue 1 may be regarded the best candidate for one of the most primitive dwarf galaxies still observable (see also $\mathrm{Ji}$ et al. 2015; Webster et al. 2016). But how common could surviving first, or very early, galaxies be today? This can only

\footnotetext{
This paper includes data gathered with the $6.5 \mathrm{~m}$ Magellan Telescopes located at Las Campanas Observatory, Chile.
}

be answered with detailed inspections of the stellar chemical abundances in additional dwarf systems. This is, however, challenging given the faint nature of even the brightest available stars near the tip of the red giant branch. Highresolution spectra are thus only available of a few stars per galaxy: e.g., three each in Ursa Major II and Coma Berenices (Frebel 2010); one star in Leo IV (Simon et al. 2010); 12 stars in Hercules, although most of them have a very limited red wavelength coverage (Koch et al. 2008; Adén et al. 2011); seven stars in Segue 1 (Norris et al. 2010a; Frebel et al. 2014); and four stars in Boötes II (Koch \& Rich 2014; Ji et al. 2016b.

Fortunately, Boötes I has been studied extensively since its discovery in 2006 by Belokurov et al. (2006). Beginning with medium-resolution spectroscopic surveys (Muñoz et al. 2006; Norris et al. 2008, 2010b), membership was established and then the $[\mathrm{Fe} / \mathrm{H}]$ distributions were determined. Hughes et al. $(2008,2014)$ found a large abundance spread of at least from $-3.7<[\mathrm{Fe} / \mathrm{H}]<-1.9$ based on photometric metallicities. $[\mathrm{C} / \mathrm{Fe}]$ spreads and $\alpha$-abundances were also spectroscopically measured for some subsamples (Lai et al. 2011). From these samples, a total of 11 stars have been observed at highresolution to produce their chemical abundance patterns (Feltzing et al. 2009; Norris et al. 2010c; Gilmore et al. 2013; Ishigaki et al. 2014). Most of these stars have been observed by more than one group. This has led to wellestablished abundances for these stars, which is of importance for the overall interpretation of the chemical inventory of Boötes I. Additionally, a kinematic study has been carried out (Koposov et al. 2011), age estimates for Boötes I have been 
Table 1

Observing Details

\begin{tabular}{lcccccccc}
\hline \hline Star & $\begin{array}{c}\alpha \\
(\mathrm{J} 2000)\end{array}$ & $\begin{array}{c}\delta \\
(\mathrm{J} 2000)\end{array}$ & UT Dates & Slit & $\begin{array}{c}t_{\text {exp }} \\
(\mathrm{hr})\end{array}$ & $\begin{array}{c}g_{0} \\
(\mathrm{mag})\end{array}$ & $\begin{array}{c}(g-r)_{0} \\
(\mathrm{mag})\end{array}$ & $\begin{array}{c}\mathrm{S} / \mathrm{N} \\
5300 \AA\end{array}$ \\
\hline Boo-980 & 135912.68 & +134255.8 & 2010 Mar 07/08/18/24 & $1^{\prime \prime \prime} 0$ & 7.0 & 18.51 & 0.61 & 25 \\
Boo-127 & 140014.57 & +143552.7 & 2011 Mar 10/12 & $11^{\prime \prime} 0$ & 3.7 & 18.15 & 0.76 & 30 \\
\hline
\end{tabular}

Note. The $\mathrm{S} / \mathrm{N}$ is measured per $\sim 33 \mathrm{~m} \AA$ pixel.

obtained (Okamoto et al. 2012; Brown et al. 2014; Hughes et al. 2014), and its chemical evolution has been modeled (Romano et al. 2015).

In this paper we report observations for one star in Boötes I with no previous high-resolution spectroscopy, and a second that has been observed before. Our goal is to investigate the chemical enrichment history of Boötes I to find out to what extent this galaxy shows signs of being a surviving first galaxy. To test such a hypothesis, we use the observational criteria suggested by Frebel \& Bromm (2012) for the metallicity spread and different abundance levels in a given system. As part of this we also aim to quantify whether there were multiple stellar generations present and to what extent chemical evolution occurred in this system. This follows earlier hints that some element contributions by Type Ia supernovae have occurred in the system (Gilmore et al. 2013). Because the luminosity of Boötes $\mathrm{I}$ is about 100 times higher than that of Segue 1, and nearly $10^{5} L_{\odot}$ (Muñoz et al. 2006), we also aim to address how the overall extent of chemical evolution relates to the luminosity of the system. Additional considerations in addition to the metallicity-luminosity relationship (e.g., Kirby et al. 2008) that appears to exist for all dwarf galaxies may shed light on the formation and evolution of these objects.

In Section 2 we describe our new observations and in Section 3 we present our analysis techniques and corresponding chemical abundance results. We apply a set of chemical abundances criteria to our and literature abundances to assess to what extent Boötes I is a surviving first galaxy in Section 4. We discuss our findings in Section 5.

\section{TARGET SELECTION AND OBSERVATIONS}

Targets were selected from Norris et al. (2008) who had taken medium-resolution $R \sim 5000$ AAOmega spectra of Boötes I stars with the Anglo-Australian Telescope. Their wide survey covered $\sim 6$ half-light radii from the center of the galaxy. Radial velocity-confirmed members were further analyzed (Norris et al. 2010b). Star Boo-1137, with $[\mathrm{Fe} / \mathrm{H}]=-3.7$, was observed with high spectral resolution for a detailed abundance analysis (Norris et al. 2010c), as were seven additional stars (Gilmore et al. 2013). Six of those stars were also observed by Ishigaki et al. (2014). The only brighter star that had not been observed was Boo-980. Being located at 3.9 half-light radii from the center it was only covered by the Norris et al. (2010b) study. In contrast, another star, Boo-127, was observed by all these studies, first by Feltzing et al. (2009) and then by all the others. Discordant abundance ratios were found which made this star interesting for re-observation. We observed these latter two Boötes I stars with the MIKE spectrograph (Bernstein et al. 2003) on the Magellan-Clay telescope in 2010 March and 2011 March. Details of the MIKE observations and photometry taken from Norris et al. (2008) are given in Table 1. MIKE spectra have nearly full optical wavelength coverage over the range 3500-9000 A. Observing conditions during these runs were mostly clear, with an average seeing of 0 !" 8 to 1 !" 0 . The 1 !" $0 \times 5^{\prime \prime}$ slit yields a spectral resolution of $\sim 22,000$ in the red and $\sim 28,000$ in the blue wavelength regime. We used $2 \times 2$ on-chip binning. Exposure times were $\sim 4$ and $7 \mathrm{hr}$, and the observations were typically broken up into 45-55 minute exposures to avoid significant degradation of the spectra by cosmic rays.

Reductions of the individual MIKE spectra were carried out with the MIKE Carnegie Python pipeline initially described by Kelson (2003). ${ }^{6}$ The orders of the combined spectrum were normalized and merged to produce final one-dimensional blue and red spectra for further analysis. The spectra have modest $\mathrm{S} /$ Ns which range from 25 to 30 at $\sim 5300 \AA$ and 37 to 43 at $\sim 6000 \AA$. Figure 1 shows representative portions of the spectra around the $\mathrm{CH} G$-band at $4313 \AA$ and the $\mathrm{Mg} b$-lines at $5170 \AA$. For comparison we also add $\mathrm{CD}-38^{\circ} 245$ with $[\mathrm{Fe} / \mathrm{H}] \sim$ -4.2 as well as Arcturus $([\mathrm{Fe} / \mathrm{H}]=-0.5)$. They bracket the metallicities of the Boötes I stars.

\section{CHEMICAL ABUNDANCE ANALYSIS RESULTS}

We measured the equivalent widths of metal absorption lines throughout the spectra using software from Casey (2014). Blending features were treated with spectrum synthesis to obtain line abundances. Table 2 lists the lines used and their measured equivalent widths and abundances for all elements, together with $3 \sigma$ upper limits for selected elements. Full details of our analysis procedure are given in Frebel et al. (2014) who analyzed the Segue 1 stars in exactly the same manner.

Using one-dimensional plane-parallel model atmospheres with $\alpha$-enhancement from Castelli \& Kurucz (2004) and the latest version of the MOOG analysis code (Sneden 1973; Sobeck et al. 2011), we computed local thermodynamic equilibrium abundances for our Boötes I stars. Final abundance ratios $[\mathrm{X} / \mathrm{Fe}]$ are obtained using the solar abundances of Asplund et al. (2009) and are listed in Table 3. Abundance uncertainties refer to the standard deviation of the line abundance measurements for each element. In the case of elements with few lines and the standard deviation resulting in very small values, we adopt a nominal minimum uncertainty of 0.1 dex.

\subsection{Stellar Parameters}

We derive stellar parameters spectroscopically from Fe I and $\mathrm{Fe}$ II lines by minimizing abundance trends and matching the $\mathrm{Fe}_{\mathrm{I}}$ to $\mathrm{Fe}$ II abundance. We follow the procedure described in Frebel et al. (2013) which adjusts the spectroscopic

\footnotetext{
6 Available at http://obs.carnegiescience.edu/Code/python
} 

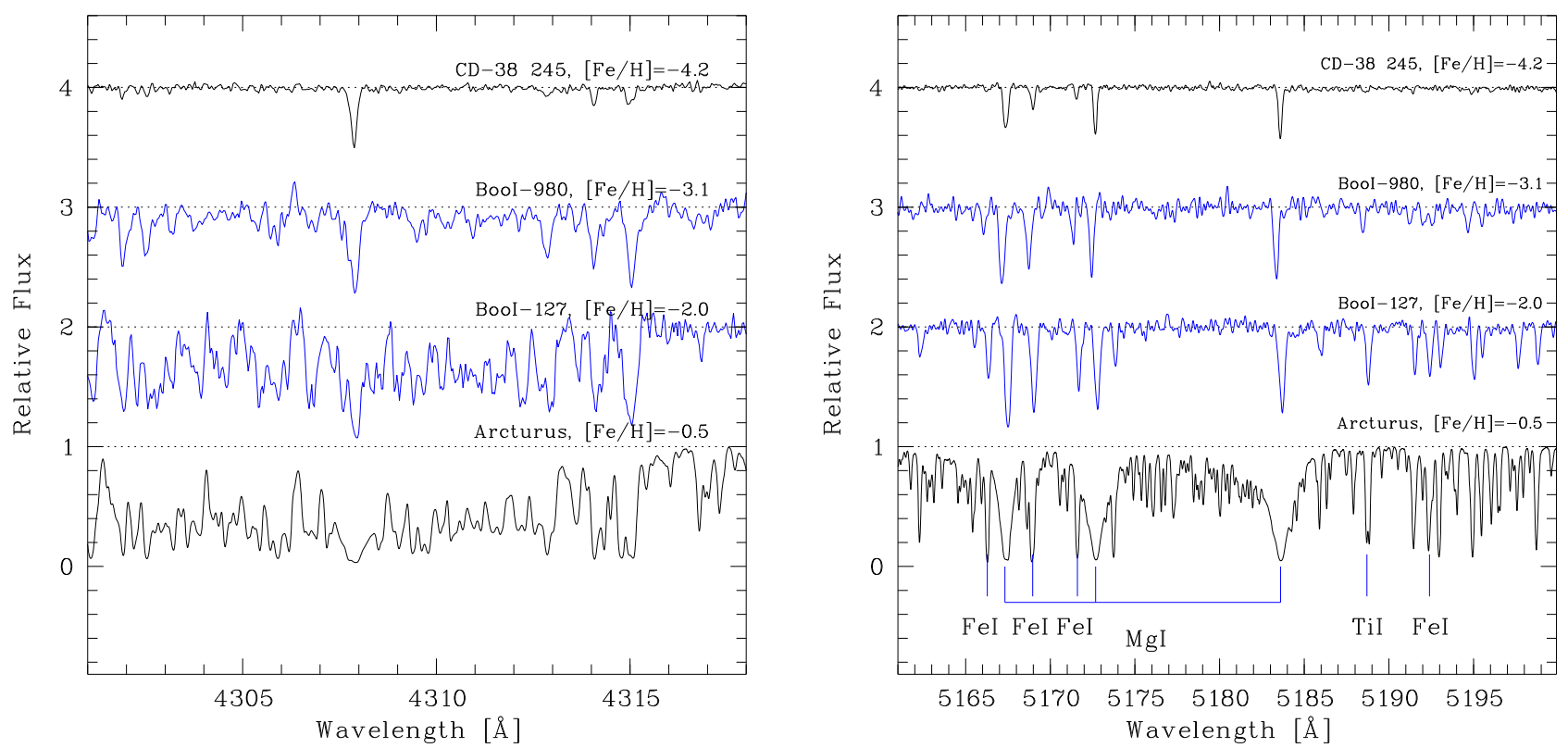

Figure 1. Magellan/MIKE spectra of our Boötes I stars, shown near the $G$-band head at $4314 \AA$ (left) and the Mg $b$-lines around $5180 \AA$ (right). Some absorption lines are indicated. The stars are bracketed in terms of their metallicity by Arcturus (bottom) and CD $-38^{\circ} 245$ (top).

Table 2

Equivalent Width Measurements of the Boötes I Stars

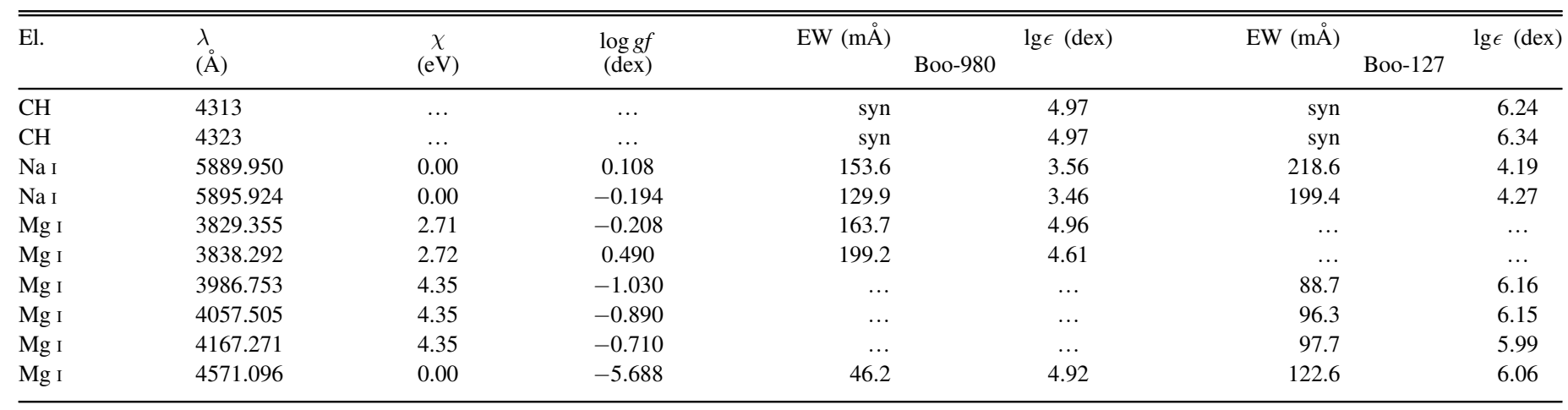

(This table is available in its entirety in machine-readable form.)

temperatures to better match those derived from photometry. In this way, no unphysically cool temperatures and low surface gravities are obtained. We note that the present analysis procedures are identical to those employed in the analysis of the stars in Segue 1 (Frebel et al. 2014). This allows us to make a homogeneous comparison between the Boötes I and Segue 1 stars. The final spectroscopic stellar parameters are $T_{\text {eff }}=$ $4720 \mathrm{~K}, \log g=1.4, v_{\text {micr }}=2.2 \mathrm{~km} \mathrm{~s}^{-1}$, and $[\mathrm{Fe} / \mathrm{H}]=-3.06$ for Boo-980 and $T_{\text {eff }}=4765 \mathrm{~K}, \quad \log g=1.35, \quad v_{\text {micr }}=$ $2.3 \mathrm{~km} \mathrm{~s}^{-1}$, and $[\mathrm{Fe} / \mathrm{H}]=-1.99$ for Boo-127. We estimate our temperature uncertainties to be $\sim 100-150 \mathrm{~K}$, and those in $\log g$ and $v_{\text {micr }}$ to be $0.3 \mathrm{dex}$ and $0.3 \mathrm{~km} \mathrm{~s}^{-1}$, respectively. The spectroscopic temperatures agree well with temperatures derived from the $(g-r)_{o}$ color transformed to $B-V$ (Jordi et al. 2006), and using color-temperature relations from Alonso et al. (1999). They are $T_{\text {eff }}=4810 \mathrm{~K}$ for Boo-980 and $T_{\text {eff }}=4625 \mathrm{~K}$ for Boo-127. Our final spectroscopic stellar parameters agree very well with values of a $12 \mathrm{Gyr}$ isochrone with $[\alpha / \mathrm{Fe}]=0.4$ and metallicities of $[\mathrm{Fe} / \mathrm{H}]=-3.0$ and -2.0 (Kim et al. 2002).

\subsection{Chemical Abundances}

Here we briefly summarize key aspects of our chemical abundance analysis. Again, we refer the reader to Frebel et al. (2014) for further details since our analysis follows the same procedures. We use the 4313 and $4323 \AA \mathrm{CH}$ features to determine the $\mathrm{C}$ abundance. Both Boo-980 and Boo-127 have slightly subsolar $[\mathrm{C} / \mathrm{Fe}]$ ratios, when taken at face value. However, one has to take into account that carbon is converted to nitrogen due to the $\mathrm{CN}$ cycle operating on the red giant branch. Many of the Boötes I stars are indeed located on the giant branch and suffer from this depletion. Calculating individual corrections based on stellar evolutionary modeling (see Placco et al. 2014 for more details) for each star to counter this effect leads to final carbon abundances of $[\mathrm{C} / \mathrm{Fe}]=0.13$ and 0.37 , for the two stars, respectively. We also apply 
Table 3

Magellan/MIKE Chemical Abundances of Our Two Boötes I Stars

\begin{tabular}{|c|c|c|c|c|c|}
\hline Species & $N$ & $\log \epsilon(\mathrm{X})$ & $\sigma$ & {$[\mathrm{X} / \mathrm{H}]$} & {$[\mathrm{X} / \mathrm{Fe}]$} \\
\hline \multicolumn{6}{|c|}{ Bоo-980 } \\
\hline $\mathrm{CH}$ & 2 & 4.97 & 0.20 & -3.46 & -0.40 \\
\hline $\mathrm{Na} \mathrm{I}$ & 2 & 3.51 & 0.10 & -2.73 & 0.33 \\
\hline Mg I & 7 & 4.83 & 0.13 & -2.77 & 0.29 \\
\hline $\mathrm{Al}$ I & 2 & 2.62 & 0.20 & -3.83 & -0.77 \\
\hline Si I & 2 & 4.88 & 0.10 & -2.62 & 0.44 \\
\hline $\mathrm{Ca} \mathrm{I}$ & 8 & 3.57 & 0.11 & -2.77 & 0.30 \\
\hline Sc II & 6 & 0.08 & 0.10 & -3.07 & -0.01 \\
\hline Ti I & 8 & 2.28 & 0.15 & -2.67 & 0.40 \\
\hline Ti II & 31 & 2.32 & 0.19 & -2.63 & 0.43 \\
\hline $\mathrm{Cr} \mathrm{I}$ & 6 & 2.13 & 0.17 & -3.51 & -0.45 \\
\hline Mn I & 3 & 1.93 & 0.10 & -3.50 & -0.44 \\
\hline $\mathrm{Fe}_{\mathrm{I}}$ & 125 & 4.44 & 0.20 & -3.06 & 0.00 \\
\hline $\mathrm{Fe}$ II & 11 & 4.46 & 0.18 & -3.04 & 0.02 \\
\hline Co I & 4 & 2.45 & 0.10 & -2.54 & 0.53 \\
\hline $\mathrm{Ni}$ I & 5 & 3.23 & 0.21 & -2.99 & 0.07 \\
\hline $\mathrm{Zn} \mathrm{I}$ & 1 & 1.90 & 0.15 & -2.66 & 0.40 \\
\hline Sr II & 2 & -1.14 & 0.20 & -4.01 & -0.95 \\
\hline Ba II & 2 & -2.11 & 0.20 & -4.29 & -1.23 \\
\hline \multirow[t]{2}{*}{ Eu II } & 1 & $<-2.44$ & $\ldots$ & $<-2.96$ & $<0.10$ \\
\hline & \multicolumn{3}{|c|}{ Boo-127 } & & \\
\hline $\mathrm{CH}$ & 2 & 6.29 & 0.20 & -2.14 & -0.15 \\
\hline $\mathrm{Na} \mathrm{I}$ & 2 & 4.23 & 0.10 & -2.01 & -0.02 \\
\hline $\mathrm{Mg}_{\mathrm{I}}$ & 6 & 6.02 & 0.11 & -1.58 & 0.41 \\
\hline $\mathrm{Al}$ I & 1 & 3.57 & 0.20 & -2.88 & -0.89 \\
\hline Si I & 1 & 6.28 & 0.20 & -1.23 & 0.76 \\
\hline $\mathrm{Ca} \mathrm{I}$ & 21 & 4.60 & 0.13 & -1.74 & 0.24 \\
\hline Sc II & 9 & 1.07 & 0.10 & -2.08 & -0.09 \\
\hline Ti I & 22 & 3.11 & 0.12 & -1.84 & 0.15 \\
\hline Ti II & 46 & 3.30 & 0.22 & -1.65 & 0.33 \\
\hline Cr I & 20 & 3.57 & 0.18 & -2.07 & -0.09 \\
\hline $\mathrm{Cr}$ II & 2 & 3.64 & 0.11 & -2.00 & -0.01 \\
\hline Mn I & 7 & 2.94 & 0.14 & -2.49 & -0.50 \\
\hline $\mathrm{Fe}_{\mathrm{I}}$ & 204 & 5.51 & 0.21 & -1.99 & 0.00 \\
\hline $\mathrm{Fe}$ II & 26 & 5.53 & 0.17 & -1.97 & 0.01 \\
\hline Co I & 4 & 2.88 & 0.16 & -2.11 & -0.12 \\
\hline Ni I & 16 & 4.17 & 0.44 & -2.05 & -0.07 \\
\hline $\mathrm{Zn}$ I & 1 & $<2.52$ & $\ldots$ & $<-2.04$ & $<-0.05$ \\
\hline $\mathrm{Sr}$ II & 1 & -0.32 & 0.99 & -3.19 & -1.20 \\
\hline Ba II & 3 & -0.53 & 0.10 & -2.71 & -0.72 \\
\hline Eu II & 1 & $<-1.37$ & $\ldots$ & $<-1.89$ & $<0.10$ \\
\hline
\end{tabular}

Note.

${ }^{a}$ Derived only from the $\lambda 4215$ line. The $\lambda 4077$ line gave a spuriously low abundance.

corrections to the adopted literature carbon abundances which range from 0 dex for the warmer giants to 0.75 dex for giants with $T_{\text {eff }}=4500 \mathrm{~K}$ (see also Section 3.3 for further discussion). The corrected carbon abundances of all stars in Boötes I together with corrected values of the comparison stars are shown in Figure 2.

As can be seen in the figure, there is a clear trend of increasing carbon abundance with decreasing $[\mathrm{Fe} / \mathrm{H}]$, very similar to what is found among halo stars (e.g., Barklem et al. 2005; Placco et al. 2014). Overall, a range in carbon abundances of 2.7 dex is present among Boötes I stars, from $-0.34<[\mathrm{C} / \mathrm{Fe}]<2.31$ (after applying the carbon correction). In fact, there are eight carbon-enhanced metal-poor (CEMP) stars $[\mathrm{C} / \mathrm{Fe}]>0.7$ (CEMP subclasses are discussed further
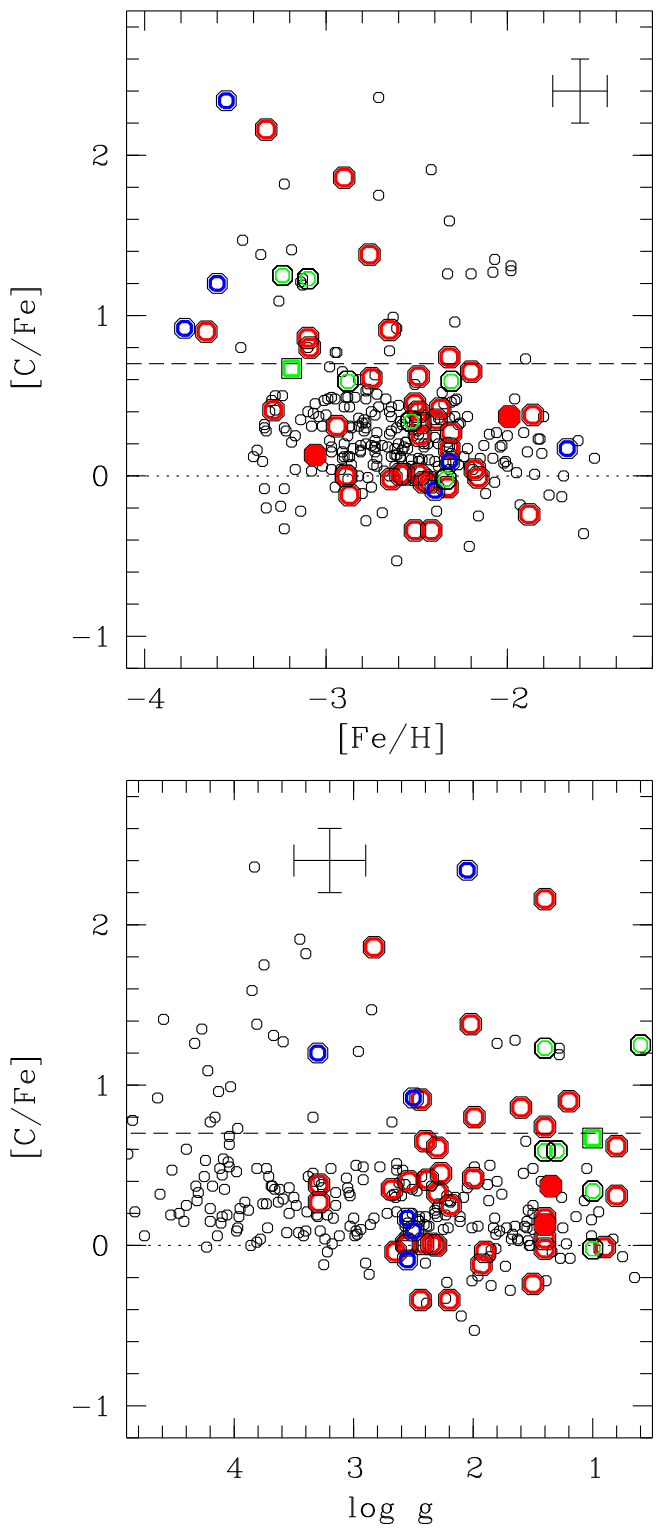

Figure 2. $[\mathrm{C} / \mathrm{Fe}]$ abundance ratios (corrected for the evolutionary status of the stars) as a function of $[\mathrm{Fe} / \mathrm{H}]$ (top panel) and surface gravity (bottom panel) of our Boötes I stars (filled red circles) and literature Boötes I stars (open red circles; Ishigaki et al. 2014 values were used for the best possible internal consistency), in comparison with metal-poor halo stars (black circles; Barklem et al. 2005) and other dwarf galaxy stars (Segue 1: blue circles (Frebel et al. 2014); UMa II, ComBer, and Leo IV: green circles (Frebel et al. 2010; Simon et al. 2010). Representative error bars are also shown. The definition of C-enhancement from Aoki et al. (2007) is shown with a dashed line.

below) after the application of the carbon corrections of Placco et al. (2014). (There are five CEMP stars before the correction.) This means that the overall fraction of CEMP stars is eight out of 39 , and thus $\sim 21 \%$. For stars with $[\mathrm{Fe} / \mathrm{H}]<-2.5$, the fraction is seven out of 19 , and thus $37 \%$. This increases to $66 \%$ (four out of six) for $[\mathrm{Fe} / \mathrm{H}]<-3.0$, and $100 \%$ (one star) for $[\mathrm{Fe} / \mathrm{H}]<-3.5$. These fractions of CEMP stars are considerably higher than the results from halo stars for the respective metallicities ranges, $24 \%, 43 \%$, and $60 \%$ (Placco et al. 2014). Note, however, that the Boötes I results are of course based on significantly smaller samples. Nevertheless, we broadly conclude that Boötes I does not show a carbon 
abundance distribution that is different from that of the Galactic halo.

Although also plagued by low number statistics, the same (qualitative) behavior is found for the combined population of Segue 1 (excluding their metal-rich $\mathrm{CH}$ star which is not shown in Figure 2), Ursa Major II, Coma Berenices, and Leo IV stars. The overall fraction is five out 13 (38\%). No stars with $[\mathrm{Fe} / \mathrm{H}]>-3.0$ are CEMP stars but the five stars with $[\mathrm{Fe} / \mathrm{H}]<-3.0$ are all carbon enhanced $(100 \%)$.

Interestingly, two of the eight carbon-enhanced stars in Boötes I for which barium abundances are available belong to the class of CEMP-no stars, that is, CEMP stars without supersolar enhancement in neutron-capture elements, i.e., $[\mathrm{Ba} / \mathrm{Fe}]<0$ (Beers \& Christlieb 2005). Additional data would be needed to determine whether the remaining six objects are also CEMP-no stars. It should be noted, however, that no star with a barium measurement in Boötes I has enhanced neutroncapture element abundances compared to the solar level, making it likely that these six objects will turn out to be CEMPno stars also. This speculation is furthermore supported by the fact that all CEMP stars in the group of ultra-faint dwarfs of Segue 1 (SDSS J100714+160154, SDSS J100652+160235, SDSS J100639+160008, Segue 1-7), Ursa Major II (UMa IIS1), and Leo IV (Leo IV-S1) stars are CEMP-no stars. For completeness, we also note that one CEMP-s star was found in Segue 1. Interestingly, several CEMP-r stars were recently found in the ultra-faint dwarf galaxy Reticulum II (DES J033523540407, DES J033607540235, DES J033454540558) whose r-process elements likely stem from a independent source than all other elements, namely a neutron star merger or magnetar (Ji et al. 2016a).

Again, this behavior is very similar to that of halo stars which adds evidence that the most metal-poor halo stars could have originated in small dwarf galaxies such as the systems discussed here (see e.g., discussion in Frebel \& Norris 2015). A further extensive discussion on the carbon abundances in Boötes I in the context of chemical evolution can be found in Gilmore et al. (2013) and will not be repeated here.

We used the Mg I, Si I, Ca I, and Ti II lines to determine $\alpha$ element abundances from equivalent width measurements and spectrum synthesis (in the case of $\mathrm{Si}$ ). Our two stars have enhanced, halo-typical $\alpha$-enhancement. Figure 3 shows the comparison of most of our elemental abundances for the two stars, in comparison with those of the extremely metal-poor star samples from Cayrel et al. (2004), Yong et al. (2013), as well as the Segue 1 ultra-faint dwarf galaxy stars (Frebel et al. 2014). Overall, the agreement of the different groups of stars is remarkably good.

Considering the full Boötes I sample, a similar behavior is found. About two-thirds of the Boötes I stars with highresolution abundance measurements have halo-typical $\alpha$-element abundances, as can be seen in Figure 4 in the top three panels. A combined $[\alpha / \mathrm{Fe}]$ versus $[\mathrm{Fe} / \mathrm{H}]$ (bottom two panels) then delivers an overall reasonable agreement with halo star abundances. No obvious downturn to solar-level ratios are found for $\mathrm{Mg}, \mathrm{Ca}, \mathrm{Ti}$, and the combined $[\alpha / \mathrm{Fe}]$, despite some individual stars having low abundances. Overall, though, the slope of $[\alpha / \mathrm{Fe}]$ does somewhat decrease with increasing $[\mathrm{Fe} / \mathrm{H}]$.

Excluding Boo-119, a CEMP-no star with $[\mathrm{Fe} / \mathrm{H}]=-3.3$ and $[\alpha / \mathrm{Fe}]=0.77$, the $[\alpha / \mathrm{Fe}]$ slope is -0.10 (rms scatter of $0.12 \mathrm{dex}$ ), based on the high-resolution abundances (second lowest panel in the figure). Also excluding Boo-41 (with
$[\mathrm{Fe} / \mathrm{H}] \sim-1.9)$, as Gilmore et al. (2013) did, increases the slope to -0.18 . Gilmore et al. found -0.19 , for comparison. When adding the combined $[\alpha / \mathrm{Fe}]$ measurements of Lai et al. (2011), the slope decreases to -0.03 (bottom panel of Figure 4; excluding only Boo-119), with an rms scatter of 0.13 dex. The implications of this behavior will be further discussed in Section 4.

Iron-peak element abundances were obtained from lines as listed in Table 2. The abundances found for our two Boötes I stars are also in excellent agreement with those of comparable halo stars (see Figure 3). This indicates the robust production of these elements in Boötes I, in the same way as in other dwarf galaxies and in the halo. This behavior thus appears to be independent of environment.

Finally, $\mathrm{Sr}$ and $\mathrm{Ba}$ are the only neutron-capture elements detectable in the two stars. Boo-980 has $\mathrm{Sr}$ and $\mathrm{Ba}$ abundances similar to those of halo stars with $[\mathrm{Fe} / \mathrm{H}] \sim-3$, although they are on the lower end of that range. They are actually rather similar to the abundances of Leo IV-SI in Leo IV studied by Simon et al. (2010) and two of the Ursa Major II stars (UMa II-S1, UMa II-S2) of Frebel (2010). This can be seen in Figure 5 , where we show both $[\mathrm{Sr}, \mathrm{Ba} / \mathrm{Fe}]$ and $[\mathrm{Sr}, \mathrm{Ba} / \mathrm{H}]$ to best illustrate the behavior of neutron-capture elements. Boo-127 shows a somewhat different behavior. Its $\mathrm{Sr}$ abundance is about 1 dex below the general $\mathrm{Sr}$ trend set by halo stars. However, based on about half a dozen data points, Boo-127 seems to extend an emerging second branch (marked in the figure) of $\mathrm{Sr}$ abundance about one dex below the main halo trend. It currently consists mainly of four Ursa Major II and Coma Berenices stars, two more Boötes I stars, together with a few halo stars. Additional measurements of $\mathrm{Sr}$ in stars with $[\mathrm{Fe} / \mathrm{H}]>-2$ in ultra-faint dwarf galaxies would help to further investigate the reality of this putative branch, in particular for Boötes I, as currently only five stars have $\mathrm{Sr}$ measurements.

Boo-1137 also has a $\mathrm{Sr}$ abundance indistinguishable from halo stars. However, its $[\mathrm{Sr} / \mathrm{H}]$ level is extremely low at $[\mathrm{Sr} / \mathrm{H}] \sim-5$. Boo-94 has an even lower value of $[\mathrm{Sr} / \mathrm{H}] \sim-5.4$. Compared to Boo-127, this indicates a $[\mathrm{Sr} /$ $\mathrm{H}$ ] spread of at least 2 dex. The low level of $\mathrm{Sr}$ abundances in these two stars is comparable with that found in Segue 1. Generally, the $\mathrm{Ba}$ abundances of all Boötes I stars are somewhat uniformly offset from the main halo star trend. The $\mathrm{Ba}$ abundance spread is $1.5 \mathrm{dex}$, and as in the case of $\mathrm{Sr}$, the lowest $\mathrm{Ba}$ abundances are similar to those found in Segue 1. This behavior will be discussed further in Section 4. Interestingly, six Boötes I stars including Boo-127 cluster around $[\mathrm{Ba} / \mathrm{H}] \sim-2.7$ and $[\mathrm{Fe} / \mathrm{H}] \sim-2.2$. These stars also have other similar abundances and could perhaps represent a dissolved star cluster candidate (Bland-Hawthorn et al. 2010; Karlsson et al. 2012) although more accurate abundances are needed to draw firm conclusions.

\subsection{Comparison of Boo-127 Abundances}

Boo-127 was first observed by Feltzing et al. (2009) who found the star to have unusually high $\mathrm{Mg}([\mathrm{Mg} / \mathrm{Fe}]=0.76)$ and low $\mathrm{Ca}([\mathrm{Ca} / \mathrm{Fe}]=0.02)$ abundances. However, these results were not confirmed by Gilmore et al. (2013) or Ishigaki et al. (2014) who also included this star in their samples. Our independent observation of Boo-127 also yields much more moderate values of $[\mathrm{Mg} / \mathrm{Fe}]=0.41$ and $[\mathrm{Ca} / \mathrm{Fe}]=0.24$, in line with the Gilmore et al. and Ishigaki et al. studies. We thus 

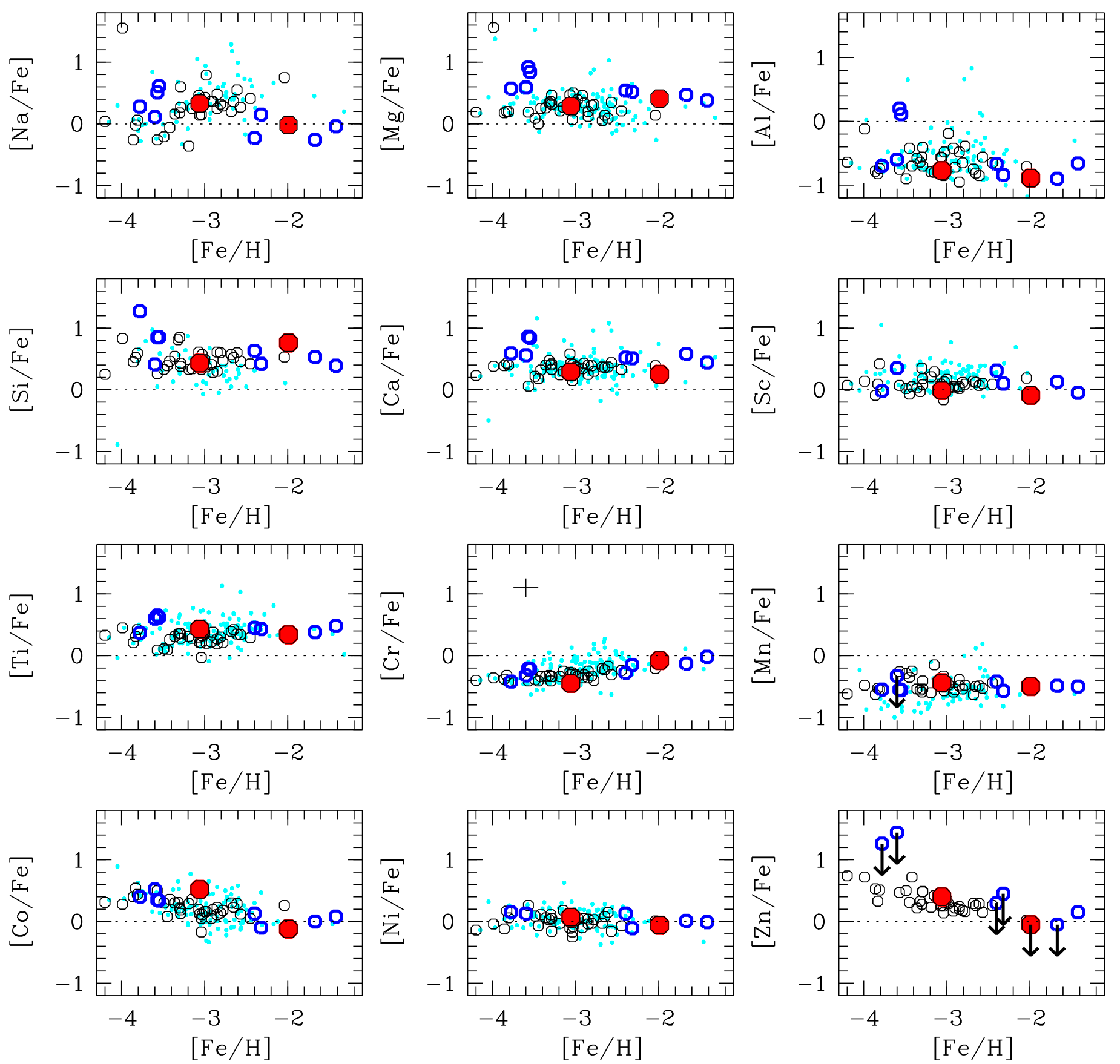

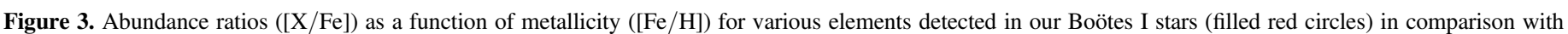

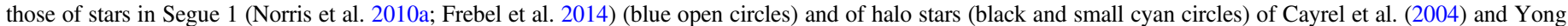
et al. (2013), respectively.

conclude that Boo-127 has no unusual $\alpha$-abundances, but rather a halo-like signature in these elements.

Figure 6 shows a detailed comparison of various elements observed in Boo-127 that are common to these four studies. The [C/Fe] abundance of Norris et al. (2010b) is also included for comparison. Except for the high Mg abundance of Feltzing et al. (2009) and the low carbon abundance of Ishigaki et al. (2014), the abundances agree reasonably well and are within $\sim 0.3$ dex of each other. We note here that Ishigaki et al. (2014) have systematically lower carbon abundances compared to our study, Norris et al. (2010b) and Lai et al. (2011) by 0.43 dex (based on five stars). We note here that the stellar parameters of all four studies are consistent, however. The effective temperatures agree within $165 \mathrm{~K}$ and the surface gravities within 0.6 dex. The $[\mathrm{Fe} / \mathrm{H}]$ abundances are remarkably close and within 0.11 dex. Excluding the Feltzing et al. (2009) results decreases these ranges to $65 \mathrm{~K}, 0.25 \mathrm{dex}$, and 0.07 dex. Since the $\mathrm{Mg} b$-lines are gravity sensitive (e.g., Hollek et al. 2011), the lower Feltzing et al. gravity choice of $\log g=1.0$ might, in retrospect, explain their high $\mathrm{Mg}$ abundance.

\subsection{Literature Data on Boötes I}

In Table 4 we list the abundances of key elements common to all studies of Boötes I stars. We list each star's name as 

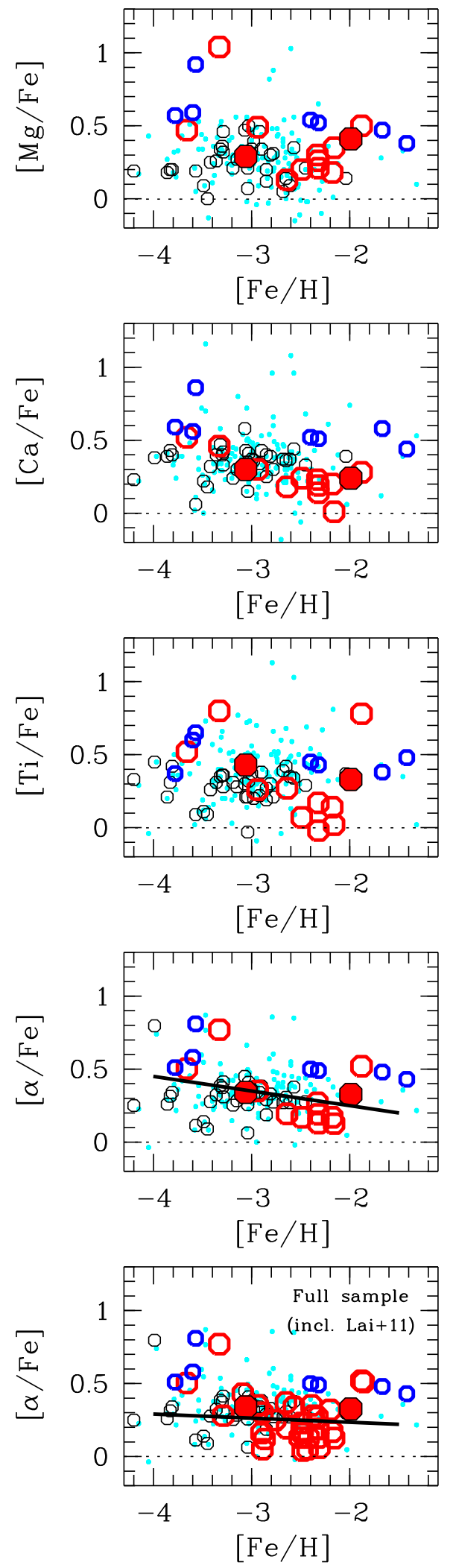

Figure 4. Abundance ratios ([X/Fe]) of $\alpha$-elements of our two Boötes I stars (full red circles) together with 11 Boötes I stars (filled red circles) as analyzed by Gilmore et al. (2013), Ishigaki et al. (2014), and Feltzing et al. (2009). Segue 1 stellar abundances (Frebel et al. 2014) are shown with blue circles, and halo stars in black circles (Cayrel et al. 2004) and cyan dots (Yong et al. 2013). The bottom two panels show combined $[\alpha / \mathrm{Fe}]$ ratios. The bottom panel additionally includes the $[\alpha / \mathrm{Fe}]$ of Lai et al. (2011). Fits to the Boötes data points (with Boo-119 excluded; $[\alpha / \mathrm{Fe}]=0.77$ ) are also shown.
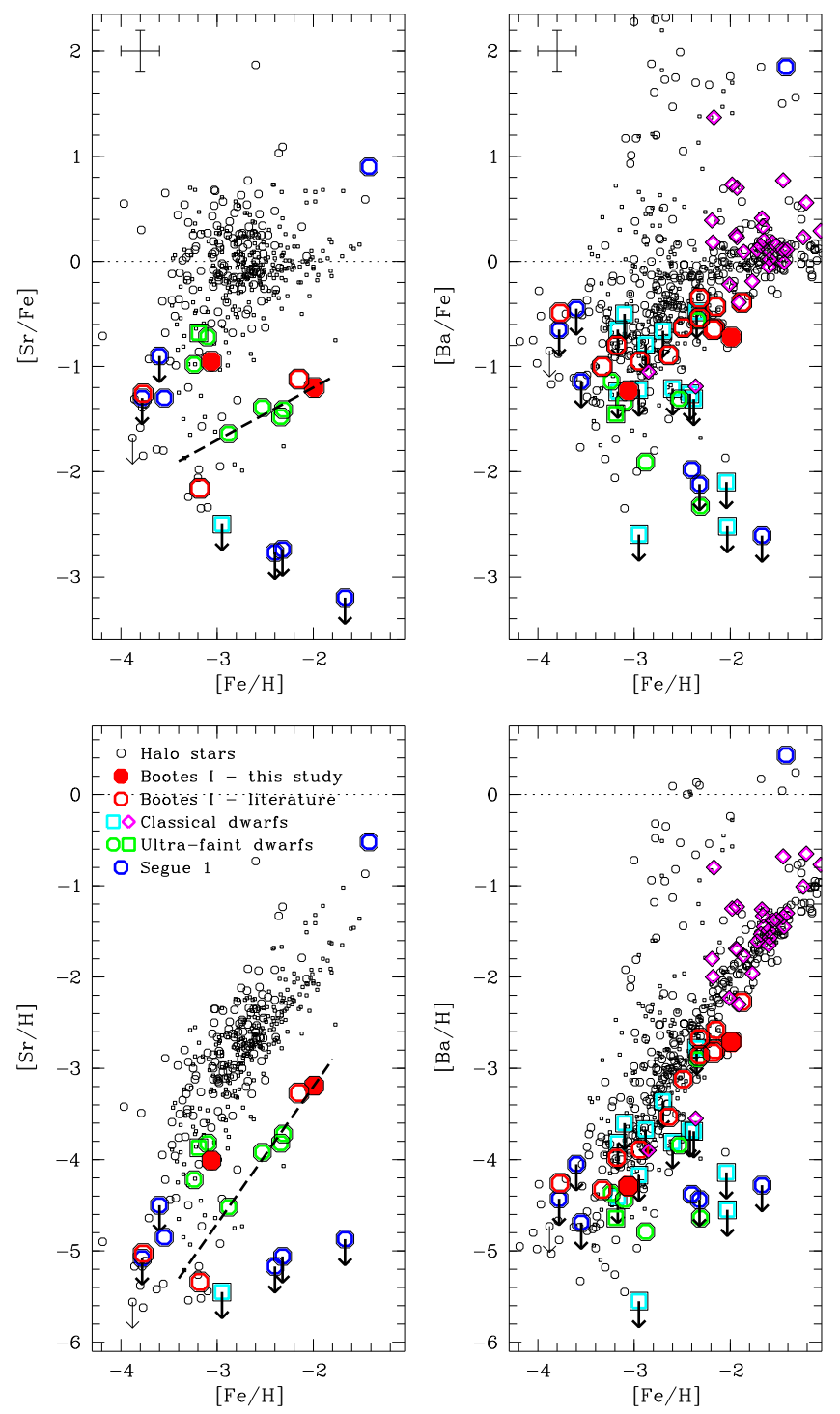

Figure 5. Abundance ratios of neutron-capture-elements $[\mathrm{Sr} / \mathrm{Fe}]$ and $[\mathrm{Ba} / \mathrm{Fe}]$ (top panels) and $[\mathrm{Sr} / \mathrm{H}]$ and $[\mathrm{Ba} / \mathrm{H}]$ (bottom panels) as a function of metallicity $[\mathrm{Fe} / \mathrm{H}]$ of our Boötes I stars (filled red circles) and literature Boötes I stars (open red circles; here Ishigaki et al. 2014 was used for Sr and Gilmore et al. 2013 for Ba, for internal consistency) in comparison with those of other ultra-faint dwarf galaxy stars in Segue 1 (blue circles; Norris et al. 2010a; Frebel et al. 2014), UMa II, ComBer, Leo IV (green circles; Frebel et al. 2010; Simon et al. 2010), Draco and Hercules (cyan squares; Fulbright et al. 2004; Koch et al. 2008, 2013), stars in the classical dwarf spheroidal galaxies (pink triangles; Venn et al. 2004), and the galactic halo (black open circles; Aoki et al. 2005; Barklem et al. 2005; François et al. 2007 and Yong et al. 2013). The dashed line is meant to guide the eye regarding a putative second dwarf galaxy star sequence. Note that both axes have the same scale, showing the huge range of neutron-capture abundances in metal-poor stars. Representative error bars are shown in the $[\mathrm{Sr} / \mathrm{Fe}]$ and $[\mathrm{Ba} / \mathrm{Fe}]$ panels.

provided by the original authors. We note that this results in an apparently inconsistent system where, e.g., two distinct stars having the names Boo-7 and Boo07. Where applicable, two distinct names for the same star are also indicated. Coordinates for these stars can be found in the references given in the last column.

For many stars, duplicate studies exist. Given authors who use different data and analysis methods, to obtain a set of abundances as internally consistent as possible between studies, 


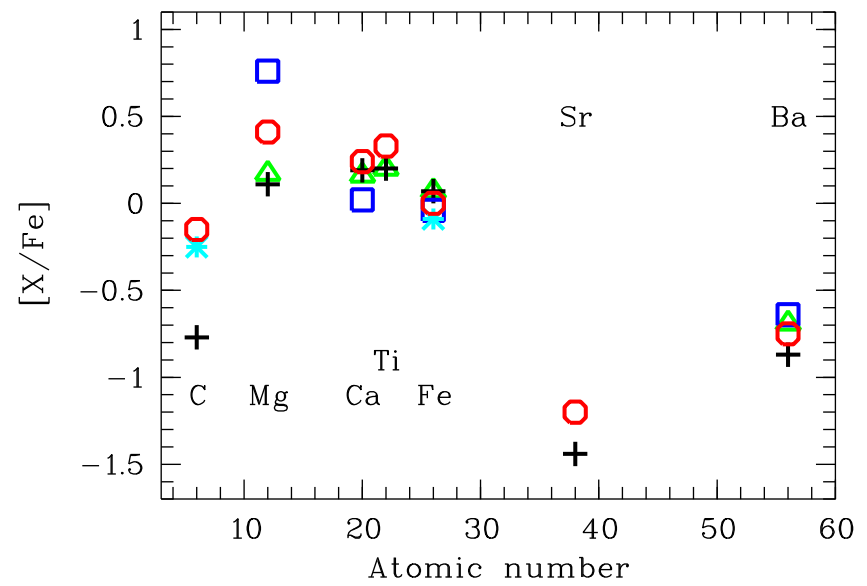

Figure 6. Abundance ratios $([\mathrm{X} / \mathrm{Fe}])$ of the four different studies that analyzed Boo-127 as a function of atomic number. In the case of iron, $[\mathrm{Fe} / \mathrm{H}]-[\mathrm{Fe} /$ $\mathrm{H}]_{\text {Boo-127, this study }}$ is shown on the $y$-axis. Symbols are as follows: red circles: this work; green triangles: Gilmore et al. (2013); black crosses: Ishigaki et al. (2014); blue squares: Feltzing et al. (2009); and cyan asterisk: Norris et al. (2010b).

and following the abundance comparison in Figure 6, we adopt a combination of (adjusted) abundances for our analysis and in the figures shown. Adjustments were made to reflect different iron abundances, e.g., $\left.[\mathrm{C} / \mathrm{Fe}]_{\text {adopted }}=[\mathrm{C} / \mathrm{Fe}]_{\text {orig }}+\Delta \mathrm{Fe} / \mathrm{H}\right]$.

Generally, we adopted our own and the Gilmore et al. (2013) abundances (we adopted an average of the "NY" and "GM" abundances) together with the carbon values from Norris et al. (2010b). We adjusted the [Sr/Fe] values from Ishigaki et al. (2014) to the Gilmore et al. scale. We replaced the carbon abundance upper limits of Ishigaki et al. (2014) with detections of Norris et al. (2010b). We used the adjusted carbon abundance of Norris et al. (2010b) together with the adopted abundances of Feltzing et al. (2009) in the case of Boo-7. Explanations of which elemental abundances have been used are given in the table notes but for clarity we list our adopted abundances for each star at the bottom of the table.

Regarding abundance uncertainties in $[\mathrm{X} / \mathrm{Fe}]$, systematic uncertainties taking into account uncertainties in the stellar parameters as well as random uncertainties are around $0.2-0.3$ dex for this kind of data quality (e.g., Table 4 in Frebel et al. 2014). Keeping this in mind, the various abundance averages are not significantly affected by our attempt to construct a homogeneous abundance set and vary by on order 0.1 dex. Nevertheless, even such subtle systematic changes could affect the interpretation, and more generally, are important for comparisons with chemical evolution models.

\section{ON THE ORIGIN AND EVOLUTION OF BOÖTES I}

Using all available abundances of stars in Boötes I, as described in the previous section, we now investigate the global chemical abundance signatures to characterize the origin and history of this ultra-faint dwarf galaxy. In particular, we use four abundance criteria developed by Frebel \& Bromm (2012) to assess to what extent chemical evolution has taken place in this system and how far removed Boötes I might be from being a surviving first galaxy, like e.g., Segue 1. The abundance criteria concern the (a) metallicity distribution function (MDF), (b) light and iron-peak element abundances, (c) $\alpha$-elements, and (d) neutron-capture elements. Under (e) we also add a discussion on the role of carbon in these early systems. All of these are discussed in detail below and applied to the body of abundance data currently available for Boötes I.

(a) Iron abundance spread and $M D F$. This criterion stipulates that a large spread of $2-3 \mathrm{dex}$ in $[\mathrm{Fe} / \mathrm{H}]$ as well as a not too steep MDF shape (especially on the low-metallicity side) would be signs of early inhomogeneous mixing. The upper panel of Figure 7 shows the MDF of Boötes I as obtained from the sample of 39 stars that includes both mediumresolution and high-resolution $[\mathrm{Fe} / \mathrm{H}]$ abundances (Feltzing et al. 2009; Norris et al. 2010b, 2010c; Lai et al. 2011; Gilmore et al. 2013; Ishigaki et al. 2014, this work). When available, a high-resolution value was used for a given star.

A spread in $[\mathrm{Fe} / \mathrm{H}]$ of 2 dex is apparent, ranging from $[\mathrm{Fe} / \mathrm{H}] \sim-3.8$ to $[\mathrm{Fe} / \mathrm{H}] \sim-1.8$. The lack of stars $[\mathrm{Fe} / \mathrm{H}]>-1.8$ (when considering high-resolution abundances where available) is typical for ultra-faint dwarf galaxies (e.g., Kirby et al. 2008; Frebel et al. 2010, 2014). The mean value for Boötes I of $[\mathrm{Fe} / \mathrm{H}]=-2.6$ based on this combined sample is in line with previous results $([\mathrm{Fe} / \mathrm{H}]=-2.3$, Belokurov et al. 2006; $[\mathrm{Fe} / \mathrm{H}]=-2.5$, Muñoz et al. 2006, Siegel 2006, Martin et al. 2007, and Norris et al. 2008; $[\mathrm{Fe} / \mathrm{H}]=-2.6$, Lai et al. 2011).

From a theoretical viewpoint, chemical inhomogeneity likely resulted in a large spread in $[\mathrm{Fe} / \mathrm{H}]$ ranging from $[\mathrm{Fe} / \mathrm{H}] \sim-4.0$ up to $\sim-1.0$ (Greif et al. 2011), without a very pronounced peak in the $[\mathrm{Fe} / \mathrm{H}]$ distribution. This behavior is found in Segue 1 (Frebel et al. 2014) which now presumably contains only those first Pop II stars. In Boötes I, a similar population of the earliest Pop II stars could have formed, with their highest metallicity stars having $[\mathrm{Fe} / \mathrm{H}] \sim-2.0$. Any additional Boötes I stars located at and near the peak region of the MDF might then be stars from the second and/or later stellar generations formed from more homogeneous gas enriched by any first/early Pop II supernovae. Still, star formation must have been rapidly quenched to prevent stars with $[\mathrm{Fe} / \mathrm{H}]>-1.8$ to form.

Regarding the MDF shape, there is a steady increase from $[\mathrm{Fe} / \mathrm{H}] \sim-4.0$ to the distinct peak of the distribution at $[\mathrm{Fe} / \mathrm{H}] \sim-2.5$. Then, there is a relatively sharp drop-off toward higher metallicities suggesting that star formation was extinguished rather abruptly, preventing the formation of stars with $[\mathrm{Fe} / \mathrm{H}]>-1.8$. The same shape of the metallicity distribution has also been found in other, more luminous dwarf galaxies (e.g., Draco and Carina, although they have higher peak metallicities; Figure 17 of Norris et al. 2010c). Since this behavior is thus not unique to Boötes I we take it as a sign of chemical evolution (as opposed to a limited chemical enrichment), although likely in its early phases given the low peak metallicity.

Lai et al. (2011) explored the nature of the Boötes I MDF with simple chemical evolution models, following Kirby et al. (2011). Taking into account our slightly more populated MDF which also contains more stars with high-resolution $[\mathrm{Fe} / \mathrm{H}]$ measurements, it appears that their leaky-box model with preenriched initial gas would best describe the low-metallicity tail of Boötes I. That model begins with pre-enriched gas of low level of $[\mathrm{Fe} / \mathrm{H}]_{0} \sim-4.0$ which is close to the assumption of pristine gas. However, it would simultaneously underpredict the very tall peak region of the MDF. We speculate that this could signify a subsequent population of stars that formed after the initial generation emerged from gas enriched by the first/ early Pop II supernovae. 
Table 4

Chemical Abundances of Key Elements of Boötes I Stars from the Literature

\begin{tabular}{|c|c|c|c|c|c|c|c|c|c|c|c|}
\hline Name & $T_{\text {eff }}$ & $\log g$ & {$[\mathrm{Fe} / \mathrm{H}]$} & {$[\mathrm{C} / \mathrm{Fe}]$} & {$[\mathrm{Mg} / \mathrm{Fe}]$} & {$[\mathrm{Ca} / \mathrm{Fe}]$} & {$[\mathrm{Ti} / \mathrm{Fe}]$} & {$[\mathrm{Sr} / \mathrm{Fe}]$} & {$[\mathrm{Ba} / \mathrm{Fe}]$} & Comment & References \\
\hline Boo02 & 5114 & 2.00 & -2.37 & 0.36 & $0.28^{\mathrm{a}}$ & $\ldots$ & $\ldots$ & $\ldots$ & $\ldots$ & med-res & LAI11 \\
\hline Boo03 & 5127 & 1.99 & -3.09 & 0.79 & $0.43^{\mathrm{a}}$ & $\ldots$ & $\ldots$ & $\ldots$ & $\ldots$ & med-res & LAI11 \\
\hline Boo04 & 5210 & 2.68 & -2.39 & 0.34 & $0.35^{\mathrm{a}}$ & $\ldots$ & $\ldots$ & $\ldots$ & $\ldots$ & med-res & LAI11 \\
\hline Boo05 & 5077 & 2.55 & -2.89 & $<0.00$ & $0.05^{\mathrm{a}}$ & $\ldots$ & $\ldots$ & $\ldots$ & $\ldots$ & med-res & LAI11 \\
\hline Boo06 & 5404 & 2.40 & -2.20 & 0.64 & $0.32^{\mathrm{a}}$ & $\ldots$ & $\ldots$ & $\ldots$ & $\ldots$ & med-res & LAI11 \\
\hline Boo07 & 5200 & 2.54 & -2.49 & 0.39 & $0.16^{\mathrm{a}}$ & $\ldots$ & $\ldots$ & $\ldots$ & $\ldots$ & med-res & LAI11 \\
\hline \multirow[t]{2}{*}{ Boo-7 } & 4800 & 1.60 & -2.32 & -0.50 & $\ldots$ & $\ldots$ & $\ldots$ & $\ldots$ & $\ldots$ & med-res & NOR10 \\
\hline & $\ldots$ & $\ldots$ & -2.33 & $\ldots$ & 0.30 & 0.23 & $\ldots$ & $\ldots$ & -0.75 & high-res & FEL09 $9^{\mathrm{b}, \mathrm{c}}$ \\
\hline Boo08 & 5178 & 2.30 & -2.48 & 0.32 & $0.18^{\mathrm{a}}$ & $\ldots$ & $\ldots$ & $\ldots$ & $\ldots$ & med-res & LAI11 \\
\hline Boo-8 & 5090 & 2.30 & -2.75 & 0.60 & $\ldots$ & $\ldots$ & $\ldots$ & $\ldots$ & $\ldots$ & med-res & NOR10 \\
\hline Boo09 & 5563 & 2.44 & -2.65 & 0.90 & $0.37^{\mathrm{a}}$ & $\ldots$ & $\ldots$ & $\ldots$ & $\ldots$ & med-res & LAI11 \\
\hline \multirow[t]{2}{*}{ Boo-9 } & 4630 & 1.10 & -2.67 & -0.55 & $\ldots$ & $\ldots$ & $\ldots$ & $\ldots$ & $\ldots$ & med-res & NOR10 \\
\hline & 4750 & 1.40 & -2.64 & $<-0.29$ & 0.13 & 0.18 & 0.27 & $\ldots$ & -0.89 & high-res & ISH $14^{\mathrm{b}, \mathrm{c}}$ \\
\hline Boo10 & 5086 & 2.42 & -2.59 & $<0.00$ & $\ldots$ & $\ldots$ & $\ldots$ & $\ldots$ & $\ldots$ & med-res & LAI11 \\
\hline Boo11 & 5199 & 2.65 & -2.43 & -0.05 & $0.05^{\mathrm{a}}$ & $\ldots$ & $\ldots$ & $\ldots$ & $\ldots$ & med-res & LAI11 \\
\hline Boo12 & 5168 & 2.19 & -2.48 & 0.24 & $0.04^{\mathrm{a}}$ & $\ldots$ & $\ldots$ & $\ldots$ & $\ldots$ & med-res & LAI11 \\
\hline Boo13 & 5631 & 2.35 & -2.49 & $<0.00$ & $0.23^{\mathrm{a}}$ & $\ldots$ & $\ldots$ & $\ldots$ & $\ldots$ & med-res & LAI1 1 \\
\hline Boo14 & 5971 & 2.56 & -2.57 & $<0.00$ & $0.34^{\mathrm{a}}$ & $\ldots$ & $\ldots$ & $\ldots$ & $\ldots$ & med-res & LAI11 \\
\hline Boo15 & 5117 & 2.31 & -2.89 & $<0.00$ & $0.30^{\mathrm{a}}$ & $\ldots$ & $\ldots$ & $\ldots$ & $\ldots$ & med-res & LAI11 \\
\hline Boo18 & 5287 & 2.27 & -2.51 & 0.44 & $0.13^{\mathrm{a}}$ & $\ldots$ & $\ldots$ & $\ldots$ & $\ldots$ & med-res & LAI11 \\
\hline Boo19 & 5141 & 2.38 & -3.29 & 0.40 & $0.28^{\mathrm{a}}$ & $\ldots$ & $\ldots$ & $\ldots$ & $\ldots$ & med-res & LAI11 \\
\hline Boo20 & 4931 & 2.44 & -2.42 & -0.35 & $0.13^{\mathrm{a}}$ & $\ldots$ & $\ldots$ & $\ldots$ & $\ldots$ & med-res & LAI11 \\
\hline Boo22 & 4866 & 1.93 & -2.87 & -0.16 & $0.12^{\mathrm{a}}$ & $\ldots$ & $\ldots$ & $\ldots$ & $\ldots$ & med-res & LAI11 \\
\hline Boo23 & 5475 & 2.83 & -2.90 & 1.86 & $0.16^{\mathrm{a}}$ & $\ldots$ & $\ldots$ & $\ldots$ & $\ldots$ & med-res & LAI11 \\
\hline Boo25 & 5141 & 2.02 & -2.76 & 1.34 & $0.25^{\mathrm{a}}$ & $\ldots$ & $\ldots$ & $\ldots$ & $\ldots$ & med-res & LAI11 \\
\hline Boo28 & 5449 & 3.29 & -2.31 & 0.27 & $0.06^{\mathrm{a}}$ & $\ldots$ & $\ldots$ & $\ldots$ & $\ldots$ & med-res & LAI11 \\
\hline Boo30 & 5449 & 3.29 & -1.86 & 0.38 & $0.51^{\mathrm{a}}$ & $\ldots$ & $\ldots$ & $\ldots$ & $\ldots$ & med-res & LAI11 \\
\hline \multirow[t]{3}{*}{ Boo-33 } & 4730 & 1.40 & -2.29 & 0.30 & $\ldots$ & $\ldots$ & $\ldots$ & $\ldots$ & $\ldots$ & med-res & NOR10 \\
\hline & 4600 & 1.00 & -2.52 & $\ldots$ & 0.69 & 0.40 & $\ldots$ & $\ldots$ & -0.40 & high-res & FEL09 \\
\hline & 4740 & 1.40 & -2.32 & $\ldots$ & 0.26 & 0.14 & -0.02 & $\ldots$ & -0.35 & high-res & GIL13 ${ }^{\mathrm{b}, \mathrm{c}}$ \\
\hline Boo-34 & 4840 & 1.60 & -3.10 & 0.55 & $\ldots$ & $\ldots$ & $\ldots$ & $\ldots$ & $\ldots$ & med-res & NOR10 \\
\hline Boo-41 & 4750 & 1.60 & -1.93 & -0.65 & $\ldots$ & $\ldots$ & $\ldots$ & $\ldots$ & $\ldots$ & med-res & NOR10 \\
\hline \multirow[t]{2}{*}{$(=$ Boo24) } & 4798 & 1.63 & -1.65 & -0.80 & $0.46^{\mathrm{a}}$ & $\ldots$ & $\ldots$ & $\ldots$ & $\ldots$ & med-res & LAI11 \\
\hline & 4750 & 1.50 & -1.88 & $\ldots$ & 0.50 & 0.28 & 0.78 & $\ldots$ & -0.39 & high-res & GIL13 $^{\mathrm{b}, \mathrm{c}}$ \\
\hline Boo-78 & 4950 & 1.90 & -2.46 & -0.15 & $\ldots$ & $\ldots$ & $\ldots$ & $\ldots$ & $\ldots$ & med-res & NOR10 \\
\hline \multirow[t]{4}{*}{ Boo-94 } & 4570 & 0.80 & -2.90 & -0.45 & $\ldots$ & $\ldots$ & $\ldots$ & $\ldots$ & $\ldots$ & med-res & NOR10 \\
\hline & 4600 & 0.50 & -2.95 & $\ldots$ & 0.47 & $\ldots$ & 0.22 & $\ldots$ & $\ldots$ & high-res & FEL09 \\
\hline & 4560 & 0.80 & -2.94 & $\ldots$ & 0.49 & 0.30 & 0.26 & $\ldots$ & -0.95 & high-res & GIL13 $3^{\mathrm{b}, \mathrm{c}, \mathrm{d}}$ \\
\hline & 4500 & 0.80 & -3.18 & $<0.25$ & 0.39 & 0.46 & 0.55 & -2.16 & -0.80 & high-res & ISH14 \\
\hline Boo-117 & 4700 & 1.40 & -2.25 & -0.30 & $\ldots$ & $\ldots$ & $\ldots$ & $\ldots$ & $\ldots$ & med-res & NOR10 \\
\hline \multirow[t]{4}{*}{$(=\mathrm{Boo} 01)$} & 4716 & 1.65 & -2.34 & -0.50 & $0.12^{\mathrm{a}}$ & $\ldots$ & $\ldots$ & $\ldots$ & $\ldots$ & med-res & LAI11 \\
\hline & 4600 & 1.00 & -2.29 & $\ldots$ & 0.22 & 0.29 & $\ldots$ & $\ldots$ & -0.46 & high-res & FEL09 \\
\hline & 4725 & 1.40 & -2.18 & $\ldots$ & 0.18 & 0.20 & 0.14 & $\ldots$ & -0.65 & high-res & GIL13 $3^{\mathrm{b}, \mathrm{c}, \mathrm{d}}$ \\
\hline & 4750 & 1.50 & -2.15 & -0.79 & 0.04 & 0.01 & 0.44 & -1.12 & -0.43 & high-res & ISH14 \\
\hline Boo-119 & 4770 & 1.40 & -3.33 & $\ldots$ & 1.04 & 0.46 & 0.80 & $\ldots$ & -1.00 & high-res & GIL13 $^{\mathrm{c}, \mathrm{e}}$ \\
\hline$(=$ Boo 21$)$ & 4775 & 1.48 & -3.79 & 2.20 & $0.27^{\mathrm{a}}$ & $\ldots$ & $\ldots$ & $\ldots$ & $\ldots$ & med-res & LAI11 \\
\hline \multirow[t]{3}{*}{ Boo-121 } & 4630 & 1.10 & -2.37 & -0.25 & $\ldots$ & $\ldots$ & $\ldots$ & $\ldots$ & $\ldots$ & med-res & NOR10 \\
\hline & $\ldots$ & $\ldots$ & -2.44 & $\ldots$ & 0.62 & 0.38 & $\ldots$ & $\ldots$ & -0.43 & high-res & FEL09 \\
\hline & 4500 & 0.80 & -2.49 & $<-0.24$ & 0.20 & 0.24 & 0.07 & $\ldots$ & -0.63 & high-res & ISH14 ${ }^{\mathrm{b}, \mathrm{c}}$ \\
\hline \multirow[t]{5}{*}{ Boo-127 } & 4670 & 1.40 & $-2.08^{\mathrm{f}}$ & -0.25 & $\ldots$ & $\ldots$ & $\ldots$ & $\ldots$ & $\ldots$ & med-res & NOR10 \\
\hline & 4600 & 1.00 & -2.03 & $\ldots$ & 0.76 & 0.02 & $\ldots$ & $\ldots$ & -0.64 & high-res & FEL09 \\
\hline & 4685 & 1.40 & -2.01 & $\ldots$ & 0.17 & 0.16 & 0.20 & $\ldots$ & -0.69 & high-res & GIL13 \\
\hline & 4750 & 1.60 & -1.92 & -0.77 & 0.11 & 0.19 & 0.20 & -1.44 & -0.87 & high-res & ISH14 \\
\hline & 4765 & 1.35 & -1.99 & -0.15 & 0.41 & 0.24 & 0.33 & -1.20 & -0.75 & high-res & this study ${ }^{\mathrm{b}}$ \\
\hline \multirow[t]{2}{*}{ Boo-130 } & 4750 & 1.40 & -2.29 & -0.40 & $\ldots$ & $\ldots$ & $\ldots$ & $\ldots$ & $\ldots$ & med-res & NOR10 \\
\hline & 4775 & 1.40 & -2.32 & $\ldots$ & 0.21 & 0.19 & 0.17 & $\ldots$ & -0.54 & high-res & GIL13 $3^{\mathrm{b}, \mathrm{c}, \mathrm{d}}$ \\
\hline \multirow[t]{3}{*}{ Boo-911 } & 4540 & 1.10 & -1.98 & -0.55 & $\ldots$ & $\ldots$ & $\ldots$ & $\ldots$ & $\ldots$ & med-res & NOR10 \\
\hline & $\ldots$ & $\ldots$ & -2.26 & $\ldots$ & 0.11 & 0.40 & $\ldots$ & $\ldots$ & -0.56 & high-res & FEL09 \\
\hline & 4500 & 0.90 & -2.16 & -0.77 & 0.35 & -0.01 & 0.02 & $\ldots$ & -0.64 & high-res & ISH14 ${ }^{\mathrm{b}}$ \\
\hline \multirow[t]{2}{*}{ Boo-980 } & 4890 & 1.70 & -3.09 & 0.00 & $\ldots$ & $\ldots$ & $\ldots$ & $\ldots$ & $\ldots$ & med-res & NOR10 \\
\hline & 4720 & 1.40 & -3.06 & -0.40 & 0.29 & 0.30 & 0.43 & -0.95 & -1.23 & high-res & this study ${ }^{\mathrm{b}}$ \\
\hline Boo-1069 & 5050 & 2.20 & -2.51 & -0.35 & $\ldots$ & $\ldots$ & $\ldots$ & $\ldots$ & $\ldots$ & med-res & NOR10 \\
\hline Boo-1137 & 4710 & 1.20 & -3.66 & 0.20 & $\ldots$ & $\ldots$ & $\ldots$ & $\ldots$ & $\ldots$ & med-res & NOR10 \\
\hline & 4700 & 1.20 & -3.66 & 0.25 & 0.47 & 0.52 & 0.52 & -1.42 & -0.59 & high-res & NOR $10 b^{b}$ \\
\hline
\end{tabular}


Table 4

(Continued)

\begin{tabular}{|c|c|c|c|c|c|c|c|c|c|c|c|}
\hline Name & $T_{\text {eff }}$ & $\log g$ & {$[\mathrm{Fe} / \mathrm{H}]$} & {$[\mathrm{C} / \mathrm{Fe}]$} & {$[\mathrm{Mg} / \mathrm{Fe}]$} & {$[\mathrm{Ca} / \mathrm{Fe}]$} & {$[\mathrm{Ti} / \mathrm{Fe}]$} & {$[\mathrm{Sr} / \mathrm{Fe}]$} & {$[\mathrm{Ba} / \mathrm{Fe}]$} & Comment & References \\
\hline \multicolumn{12}{|c|}{ Adopted abundances of high-resolution spectroscopic studies } \\
\hline Boo-7 & $\ldots$ & $\ldots$ & -2.33 & $-0.49\left(0.07^{\mathrm{g}}\right)$ & 0.30 & 0.23 & $\ldots$ & $\ldots$ & -0.75 & high-res & FEL09, NOR10 \\
\hline Boo-9 & 4750 & 1.40 & -2.64 & $-0.58\left(-0.02^{\mathrm{g}}\right)$ & 0.13 & 0.18 & 0.27 & $\ldots$ & -0.89 & high-res & ISH14, NOR10 \\
\hline Boo-33 & 4740 & 1.40 & -2.32 & $0.29\left(0.74^{\mathrm{g}}\right)$ & 0.26 & 0.14 & -0.02 & $\ldots$ & -0.35 & high-res & GIL13, NOR10 \\
\hline Boo-41 & 4750 & 1.50 & -1.88 & $-0.78\left(-0.24^{\mathrm{g}}\right)$ & 0.50 & 0.28 & 0.78 & $\ldots$ & -0.39 & high-res & GIL13, NOR10 \\
\hline Boo-94 & 4560 & 0.80 & -2.94 & $-0.44\left(0.31^{\mathrm{g}}\right)$ & 0.49 & 0.30 & 0.26 & -2.43 & -0.95 & high-res & GIL13, NOR10, ISH14 \\
\hline Boo-117 & 4725 & 1.40 & -2.18 & $-0.50\left(0.04^{\mathrm{g}}\right)$ & 0.18 & 0.20 & 0.14 & -1.22 & -0.65 & high-res & GIL13, NOR10, ISH14 \\
\hline Boo-119 & 4770 & 1.40 & -3.33 & $1.85\left(2.16^{\mathrm{g}}\right)$ & 1.04 & 0.46 & 0.80 & $\ldots$ & -1.00 & high-res & GIL13, LAI11 \\
\hline Boo-121 & 4500 & 0.80 & -2.49 & $-0.13\left(0.62^{\mathrm{g}}\right)$ & 0.20 & 0.24 & 0.07 & $\ldots$ & -0.63 & high-res & ISH14, NOR10 \\
\hline Boo- 127 & 4765 & 1.35 & -1.99 & $-0.15\left(0.37^{\mathrm{g}}\right)$ & 0.41 & 0.24 & 0.33 & -1.20 & -0.75 & high-res & FRE14 \\
\hline Boo- 130 & 4775 & 1.40 & -2.32 & $-0.41\left(0.17^{\mathrm{g}}\right)$ & 0.21 & 0.19 & 0.17 & $\ldots$ & -0.54 & high-res & GIL13, NOR10 \\
\hline Boo-911 & 4500 & 0.90 & -2.16 & $-0.77\left(-0.01^{\mathrm{g}}\right)$ & 0.35 & -0.01 & 0.02 & $\ldots$ & -0.64 & high-res & ISH14 \\
\hline Boo-980 & 4720 & 1.40 & -3.06 & $-0.40\left(0.13^{\mathrm{g}}\right)$ & 0.29 & 0.30 & 0.43 & -0.95 & -1.23 & high-res & FRE14 \\
\hline Boo-1137 & 4700 & 1.20 & -3.66 & $0.25\left(0.90^{\mathrm{g}}\right)$ & 0.47 & 0.52 & 0.52 & -1.42 & -0.59 & high-res & NOR10b \\
\hline
\end{tabular}

Notes. Note the different numbering schemes, e.g., Boo07 and Boo-7 do not refer to the same star. Different designations for the same star are indicated.

a These values are $[\alpha / \mathrm{Fe}]$, not $[\mathrm{Mg} / \mathrm{Fe}]$.

b Abundances from this study have been adopted for this star.

${ }^{c}[\mathrm{C} / \mathrm{Fe}]$ values of Norris et al. (2010b), adjusted for the different $[\mathrm{Fe} / \mathrm{H}]$ abundances, were used.

$\mathrm{d}[\mathrm{Sr} / \mathrm{Fe}]$ values of Ishigaki et al. (2014), adjusted for the different $[\mathrm{Fe} / \mathrm{H}]$ abundances, were used.

e $[\mathrm{C} / \mathrm{Fe}]$ values of Lai et al. (2011), adjusted for the different $[\mathrm{Fe} / \mathrm{H}]$ abundances, was used.

${ }^{\mathrm{f}}[\mathrm{Fe} / \mathrm{H}]$ from Norris et al. (2010b) is based on early analysis of a high-resolution UVES spectrum. The medium-resolution value is [Fe/H] $=-1.49$.

${ }^{g}$ Final carbon abundances corrected for evolutionary status of the star (following Placco et al. 2014) is given in parenthesis.

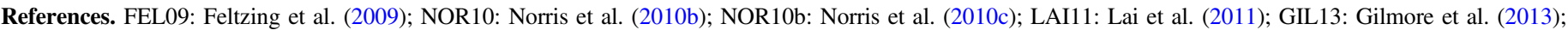

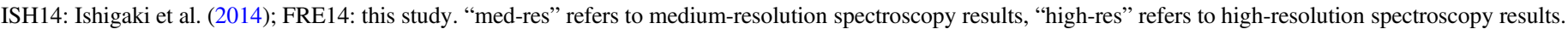

(b) Core-collapse supernova signatures. This criterion stipulates that light elements (including iron-peak elements) observed in early dwarf galaxy stars should match those of equivalent metal-poor halo stars as core-collapse supernovae are believed to be the progenitors of these elements. As can be seen in Figure 3, the observed abundances of stars in Boötes I are in good agreement with metal-poor halo star abundances, in line with a fast enrichment in the earliest dwarf galaxies.

(c) $\alpha$-element abundances: late-time star formation? This criterion stipulates that the $\alpha$-elements $(\mathrm{Mg}, \mathrm{Si}, \mathrm{Ca}, \mathrm{Ti})$ should show enhanced abundances of $\sim 0.4$ dex due to core-collapse supernova as progenitors for all stars, even at high(er) metallicities $([\mathrm{Fe} / \mathrm{H}]>-2.0)$ since it implies that no stars formed after (any) late-time enrichment of iron by Type Ia supernovae. This would support that only one/few generations of early Pop II stars formed in a system.

As noted by previous author (e.g., Gilmore et al. 2013), the $\alpha$-element abundances of stars in Boötes I do not provide a clear picture. Consideration of the individual $\alpha$-elements, e.g., in Figure 4, shows that all stars have $[\mathrm{Mg} / \mathrm{Fe}]$ high-resolution abundance ratios in agreement with halo stars. For $[\mathrm{Ca} / \mathrm{Fe}]$ and $[\mathrm{Ti} / \mathrm{Fe}]$, most Boötes I stars have typical halo values but there are also several outliers with abundance near the solar ratio. However, in all cases, the 2-3 most metal-rich stars do not have the lowest abundances but rather halo-like values. In fact, the highest metallicity star (Boo-41) has one of the highest $\alpha$-element abundances $([\alpha / \mathrm{Fe}]=0.52)$. This supports Boötes I containing those early Pop II stars, although not exclusively.

The combined $\alpha$-element abundances are then helpful to assess whether Boötes I experienced late time, extended star formation as evidenced by low(er) $\alpha$-abundances. This is illustrated in Figure 8. In the bottom two panels, we show the Boötes I abundances together with abundances of other ultrafaint dwarf galaxies as well as those of stars in the classical dwarf spheroidal galaxies. Several Boötes I stars have abundances resembling those of the halo, Segue 1, and other ultra-faint dwarf galaxy stars, while many others have values very similar to those of the classical dwarf galaxy stars (21 have $[\alpha / \mathrm{Fe}]<0.3$ and $16[\alpha / \mathrm{Fe}]<0.2)$.

It thus appears like Boötes I is a system that was assembled from or absorbed one (or a few) smaller building block type objects (e.g., minihalos) like Segue 1 before forming more stars and experiencing a chemical evolution (as opposed to just chemical enrichment as, e.g., in Segue 1) that would eventually lead to stars with low $\alpha$-abundances. Such a two-population scenario is qualitatively in line what was found based on the CEMP and non-CEMP star MDF components of Boötes I. More precise $\alpha$-element abundances will be needed for all stars (e.g., recall that Lai et al. 2011 only provided a combined $\alpha$-measurement) to conclusively determine whether any latetime enrichment by Type Ia supernovadid indeed occur in Boötes I. This would indicate extended star formation possibly due to gas accretion from other clouds or cloud fragments in the system or an early merger with a different system that would have brought in extra gas (Smith et al. 2015).

(d) Low and unusual neutron-capture element abundances: evidence for one progenitor generation? This criterion stipulates that abundances of heavy neutron-capture elements (e.g., $\mathrm{Sr}$ and $\mathrm{Ba}$ ) should be very low since it implies that no stars formed after late(r) time enrichment associated with the s-process operating in asymptotic giant branch (AGB) stars. Small amounts of neutron-capture elements, primarily with $[\mathrm{Sr} / \mathrm{Ba}]$ ratios characteristic of the r-process (occurring in supernovae) could, however, be present in the system without implying late-time star formation.

We have already discussed that the neutron-capture elements $\mathrm{Sr}$ and $\mathrm{Ba}$ are of low abundance in Boötes I (Figure 5). Specifically, the $\mathrm{Sr}$ abundances are not like those of halo stars 

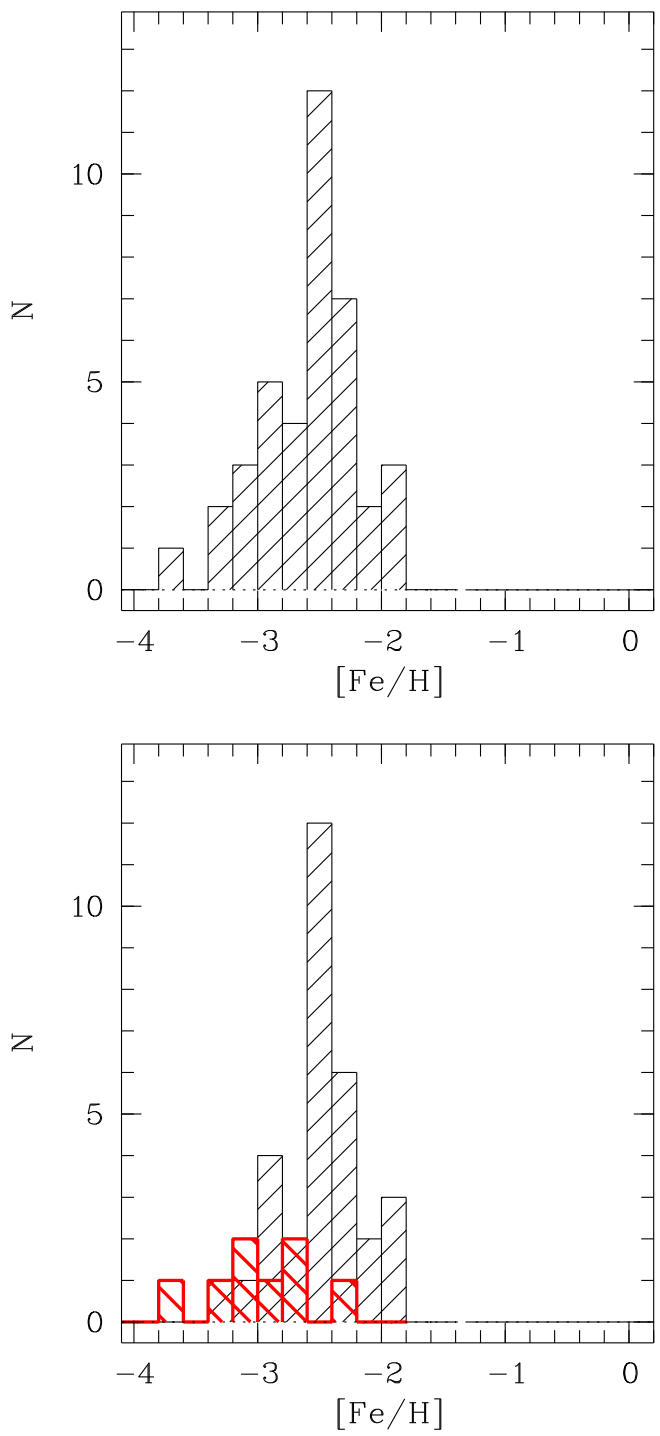

Figure 7. Metallicity distribution function for Boötes I. Note the lack of metalrich stars above $[\mathrm{Fe} / \mathrm{H}]=-1.8$. Top panel: complete sample of 39 stars. Bottom panel: red histogram depicts CEMP stars with $[\mathrm{C} / \mathrm{Fe}]>0.7$, black histogram the remaining non-CEMP stars. See part (e) of this section for additional discussion about the CEMP histogram.

but up to $\sim 1$ dex lower. Ba does roughly follow the halo star abundance trend but all Boötes I stars still have abundances that place them at the lower edge of the region covered by halo data. In addition, the spread in neutron-capture element abundances in Boötes I, as evidenced by only four stars, is about 1 dex. This precludes overarching statements regarding the exact neutron-capture process responsible for this material. The stars with $[\mathrm{Sr} / \mathrm{Ba}] \sim-0.5$ ratio technically fall in the regime of the r-process (we note that most Segue 1 stars are also near this value). The s-process is characterized by $[\mathrm{Sr} / \mathrm{Ba}]<-1.0$ and indeed one Boötes I star has such a low [Sr/Ba] value. Overall, this is consistent with Boötes I being an old system that formed its stars early and relatively quickly, before the onset of any regular AGB star-based s-process element enrichment. Consequently, fast neutron-capture enrichment might have occurred by just one of the earliest generations of massive stars in the system.

In Figure 9, there exists no obvious correlation between [Sr/ $\mathrm{Ba}]$ and $[\mathrm{Fe} / \mathrm{H}]$. When plotting $[\mathrm{Sr} / \mathrm{Ba}]$ versus $[\mathrm{Ba} / \mathrm{Fe}](\mathrm{see}$
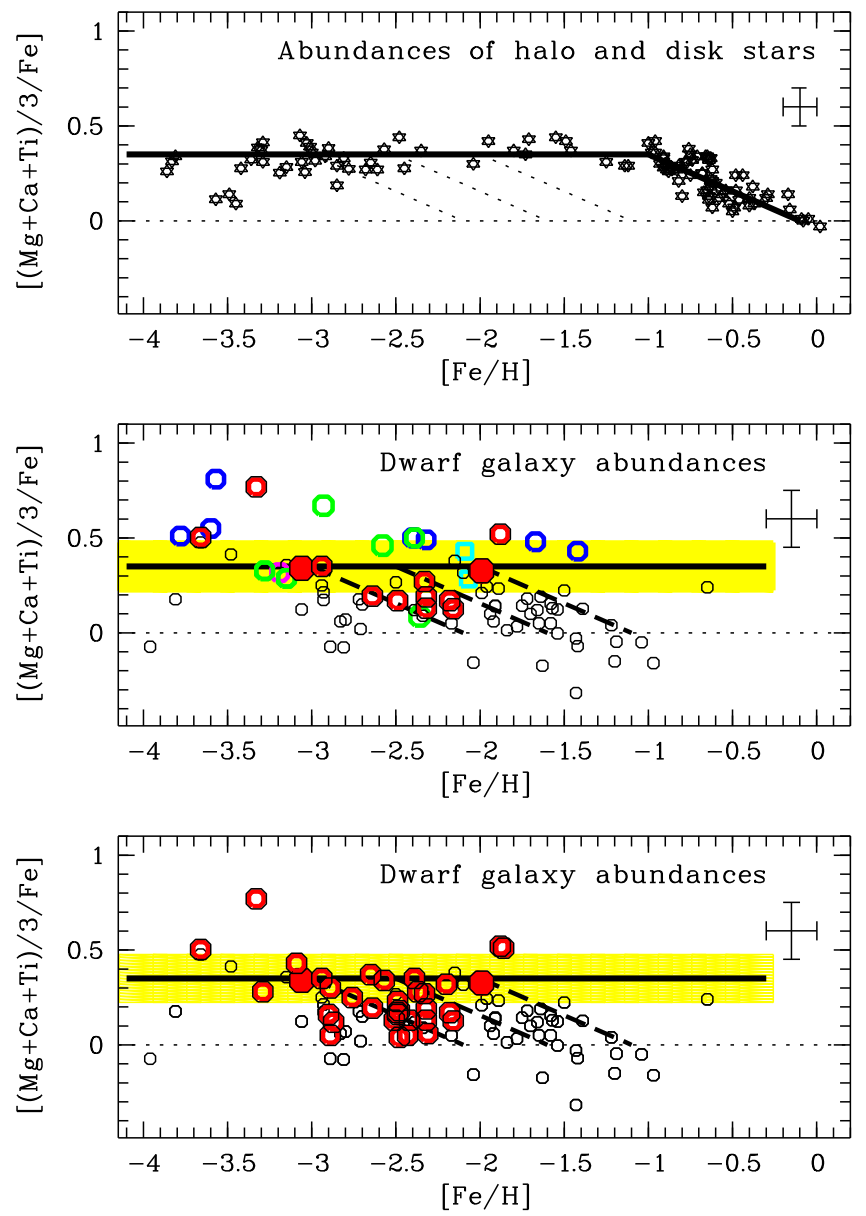

Figure 8. Combined $\mathrm{Mg}-\mathrm{Ca}-\mathrm{Ti} \alpha$-element abundances as diagnostic of early star formation; adapted from Frebel \& Bromm (2012). Top: schematic representation of chemical enrichment in the $[\alpha / \mathrm{Fe}]$ vs. $[\mathrm{Fe} / \mathrm{H}]$ plane for different environments. The dotted line indicates the solar ratio. Second: highresolution $\alpha$-abundances of metal-poor stars from Cayrel et al. (2004) (halo) and Fulbright (2000) (thin/thick disk). The diagonal dotted lines indicate the enrichment behavior of the $\mathrm{dSph}$ galaxies (see top panel). A representative uncertainty is shown. Third: the yellow shaded region around $[\alpha / \mathrm{Fe}]=0.35$ depicts the predicted Frebel \& Bromm (2012) one-shot enrichment behavior (with a 0.15 dex observational uncertainty) reflecting massive core-collapse supernova enrichment. Several evolutionary paths are indicated with dashed lines. High-resolution $\alpha$-abundances of metal-poor stars are shown; For ultrafaint dwarf galaxies: Boötes I stars, this study (filled red circles); Boötes I, literature (open red circles, see the text for references); Segue 1 (blue circles; Norris et al. 2010a; Frebel et al. 2014); Ursa Major II and Coma Berenices (green circles; Frebel 2010); Leo IV (pink circle; Simon et al. 2011); and Hercules (cyan squares; Koch et al. 2008). For classical dSphs (small open black circles; see Frebel \& Bromm 2012 and references therein). Bottom: same as above but with only the Boötes I stars that include the $[\alpha / \mathrm{Fe}]$ measurements of Lai et al. (2011).

bottom panel), however, a clear trend emerges. Interestingly, the halo stars form a distinct branch while the various dwarf galaxy stars form something that resembles a second sequence below the halo branch. The few Boötes I stars with measured $\mathrm{Sr}$ abundances follow this trend set by the UMa II, ComBer, Leo IV, and three Segue 1 stars. Some halo stars do exhibit similar neutron-capture element characteristics to those of the dwarf galaxy stars. This might indicate that these halo stars are stars that originated in small dwarf galaxies similar to those in the surviving ultra-faint dwarfs. While more data for ultra-faint dwarf galaxy stars is clearly needed to more firmly establish this second sequence, perhaps also more halo data could help 

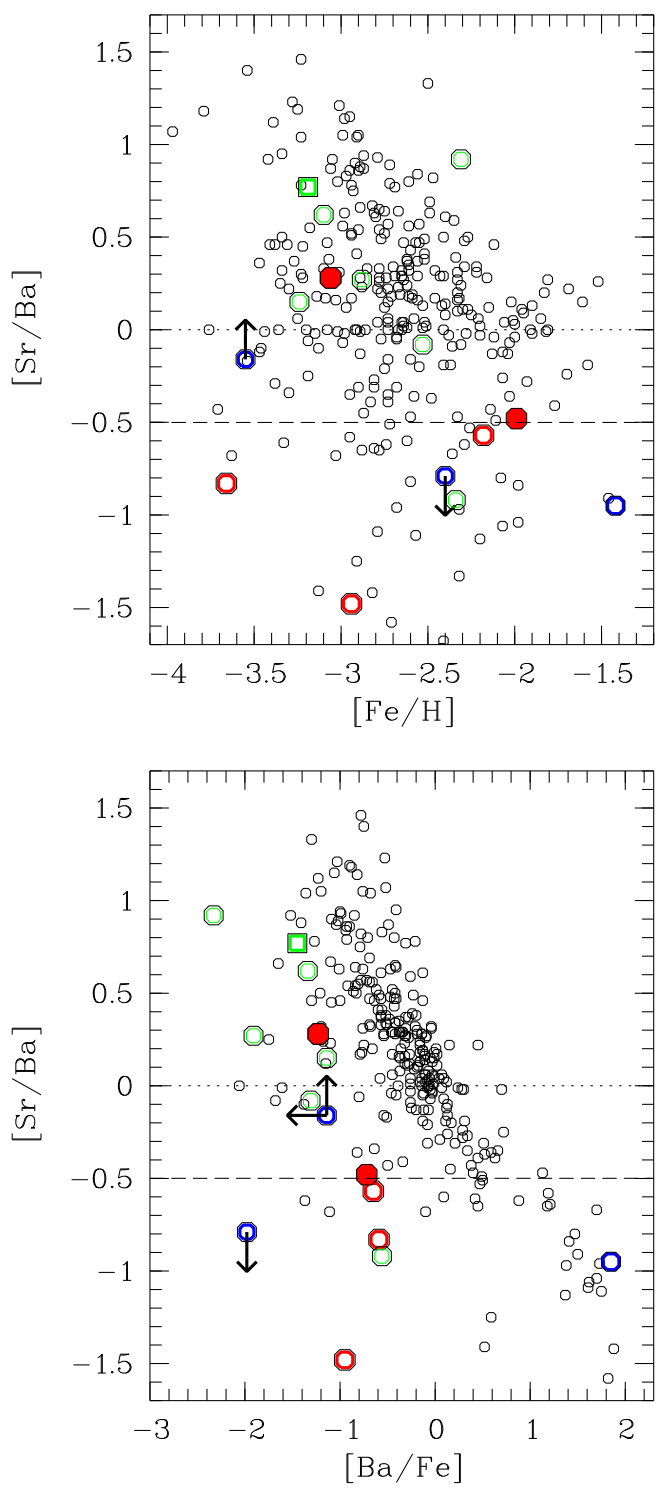

Figure 9. $[\mathrm{Sr} / \mathrm{Ba}]$ abundance ratios as a function of $[\mathrm{Fe} / \mathrm{H}]$ (top panel) and $[\mathrm{Ba} / \mathrm{Fe}]$ (bottom panel) of our Boötes I stars (filled red circles) and literature Boötes I stars (open red circles; here the Ishigaki et al. 2014 values were used for internal consistency) in comparison with those of other ultra-faint dwarf galaxy stars in Segue 1 (blue circles; Norris et al. 2010a; Frebel et al. 2014), UMa II, ComBer, Leo IV (green circles; Frebel et al. 2010; Simon et al. 2010), and the galactic halo (black open circles; Barklem et al. 2005 and Yong et al. 2013).

shape up any of the differences between the halo star branch and the dwarf galaxy sequence even more clearly.

Using neutron-capture element abundances is clearly a promising way path to learn not only about the nature of the host dwarf galaxy but also about the origin of halo stars. Lighter element abundances have been repeatedly shown to all agree with each other for halo and dwarf galaxy stars making it more challenging to use them as a discriminator.

Alternatively, the distinct patterns of halo stars and dwarf galaxy stars could originate from highly inhomogeneous enrichment of their respective birth gas clouds. However, while this would explain the large spreads in [element/H] abundance ratios such as $[\mathrm{Fe} / \mathrm{H}]$ or $[\mathrm{Sr} / \mathrm{H}]$, [element/element] ratios should in principle be hardly affected (Frebel \& Bromm 2012). Indeed, the lighter elements show tight
[element/Fe] ratios and support this possibility. In turn, the large spread in $[\mathrm{Sr} / \mathrm{Ba}]$ might indicate that the two elements are not produced with a strong correlation between them as the fusion-produced elements are, or that metal mixing did not play a primary role.

(e) Carbon enrichment: prevalent at early times? After applying carbon abundance corrections according to stellar evolutionary status (see Table 4), there are eight CEMP stars in Boötes I with metallicities below $[\mathrm{Fe} / \mathrm{H}] \lesssim-2$.6. Incidentally, the CEMP stars are all CEMP-no stars and rather equally distributed among the lower metallicity half of all Boötes I stars. All stars have $[\mathrm{C} / \mathrm{H}]$ values higher than $[\mathrm{C} / \mathrm{H}]=-3.4$ placing them well above the critical carbon and oxygen abundance criterion of $D_{\text {trans }}=\log \left(10^{[\mathrm{C} / \mathrm{H}]}+0.3 \times 10^{[\mathrm{O} / \mathrm{H}]}\right)=-3.5$ that may have facilitated their formation from carbon and oxygen enriched gas in the early universe (Frebel et al. 2007). Carbon must thus have played an important role in the early evolution of Boötes I.

$[\mathrm{C} / \mathrm{H}]$ ratios of the Boötes I sample do not show a clear trend with either $[\mathrm{Fe} / \mathrm{H}]$ or $\log g$, although the sample does follow the overall carbon abundance range given by halo stars (see Figure 2, also for references). The CEMP stars in Boötes I increasingly appear all at lower metallicities, at $[\mathrm{Fe} / \mathrm{H}] \leqslant-2.6$. Generally, the high-carbon outliers may indicate that the production of carbon and iron and their subsequent mixing into the gas has been decoupled or that CEMP and non-CEMP signatures have been produced at different sites. For a more extended discussion on the origin of CEMP stars, we refer the reader to Norris et al. (2013) or Frebel \& Norris (2015).

Cooke \& Madau (2014) suggested a minihalo environment as a site for CEMP star formation with faint fall-back first supernovae (e.g., Umeda \& Nomoto 2003) responsible for large quantities of lighter elements such as carbon but for less or none of the heavier ones like iron. The bottom panel of Figure 7 shows the separate MDFs for CEMP stars (red) and non-CEMP stars (black). The CEMP stars cluster at low metallicities albeit with some spread in $[\mathrm{Fe} / \mathrm{H}]$. But chemical inhomogeneity could possibly account for this (e.g., Frebel \& Bromm 2012). These CEMP stars thus might have formed in a minihalo environment that would later become Boötes I or part of Boötes I.

The non-CEMP star MDF lacks the pronounced lowmetallicity tail and an average metallicity of $[\mathrm{Fe} / \mathrm{H}]=-2.5$. This leaves the MDF to be more like the one predicted by the leaky-box chemical evolution model with extra gas (Kirby et al. 2011; Lai et al. 2011). This incorporates an additional reservoir of gas available to the galaxy from which to form stars. A merger of said minihalo with another early halo (presumably prior to it forming any stars) could have provided this gas. These findings build in some way on the conclusions of Gilmore et al. (2013) who suggest that the "CEMP-no" stars (our "CEMP stars") formed rapidly and prior to the "normal branch" (our "non-CEMP" stars).

More generally, massive rotating Pop III "spinstars" (Meynet et al. 2006; Chiappini et al. 2011) could have dominated the enrichment of Boötes I at the earliest times by providing large amounts on carbon to the star forming gas prior to their explosions. Fall-back supernovae (Umeda \& Nomoto 2003) could have been equally responsible for high $[\mathrm{C} / \mathrm{Fe}]$ ratios in environments more massive than minihalos. Regardless, the earliest low-mass stars would likely show a variety of $[\mathrm{C} / \mathrm{Fe}]$ 
ratios, and a clear trend would only emerge with the onset of more regular Pop II supernovae and extended star formation.

\section{CONCLUSION}

We have studied the stellar chemical abundances of Boötes I with high-resolution spectroscopy, including a previously unobserved member star. Considering all available abundance data, we examined Boötes I in light of several criteria for identifying the earliest galaxies. The $\alpha$ - and carbon abundances suggest two potential populations, although more precise abundances are required to draw firm conclusions. Considerations of these chemical signatures alone suggests that Boötes I is not a surviving first galaxy but an already somewhat assembled system that could have been built up from a minihalo (a first galaxy) in which the CEMP stars formed (Cooke \& Madau 2014), and then merged with another metalfree or metal-poor halo (or large gas clouds or filaments) that resulted in an injection of fresh gas triggering star formation and leading to a second population of stars. Both populations show $[\mathrm{Fe} / \mathrm{H}]$ spreads of $>1$ dex which is in line with predictions of chemical inhomogeneity (e.g., Frebel \& Bromm 2012) and expected for such early star formation.

Similar results have also been derived by Romano et al. (2015) who modeled the formation and chemical evolution of Boötes I. They conclude that Boötes I formed from accretion of a baryonic mass $M_{b} \sim 10^{7} M_{\odot}$ of gas over a very short timescale of order $50 \mathrm{Myr}$. This is also in agreement with what was already qualitatively predicted by Gilmore et al. (2013) who found that the CEMP stars formed rapidly and prior to the non-CEMP stars.

All these findings are highly consistent with results based on deep Hubble Space Telescope photometry. Brown et al. (2014) found Boötes I to currently consist of two ancient stellar populations of $13.4 \mathrm{Gyr}$ (containing $3 \%$ of stars) and $13.3 \mathrm{Gyr}$ ( $97 \%$ of stars). We note, however, that these ages are relative to the globular cluster M92, and assuming its age to be $13.7 \mathrm{Gyr}$. Nevertheless, it is apparent that all the dwarf galaxies contain one ancient dominant stellar population plus a small age spread. The two-population scenario follows Koposov et al. (2011) who also found that Boötes I is best described with two components, based on a kinematic analysis. They identified a "colder" $\left(\sim 3 \mathrm{~km} \mathrm{~s}^{-1}\right)$ component (containing $70 \%$ of stars) and a "hotter" $\sim 9 \mathrm{~km} \mathrm{~s}^{-1}$ component (30\% of stars). In addition, they suggested that the colder component has "an extremely extended, very metal-poor, low-velocity dispersion component". The chemical abundances and the MDF available for Boötes I stars clearly show the existence of such a lowmetallicity tail (see Figure 7) which is mainly composed of CEMP stars.

In summary, evidence based on multiple independent approaches suggests Boötes I to be an ancient system with two stellar populations of which at least the more metal-poor one likely consisted of the first low-mass stars that formed in its progenitor halo(s). This makes Boötes I an assembled system with a more complex evolution than what would be expected of a surviving galactic building block, such as Segue 1. Nevertheless, Boötes I is likely one of the earliest galaxies to have formed. Not just for its age of $13.4 \mathrm{Gyr}$ (Brown et al. 2014) but also because we seem to be witnessing what is the product of two systems, one minihalo and one larger halo which merged to form Boötes I. To some extent this outcome is already indicated by its larger luminosity of nearly $\sim 10^{5} L_{\odot}$, compared to e.g., Segue 1 with only about $\sim 10^{3} L_{\odot}$. This leaves the question as to whether an average metallicity of an early galaxy is a useful quantify if more than one population contributes stars in different metallicity ranges. Ideally, one could cleanly disentangle the population(s) to report separate results. This might work for Boötes I but will surely become impossible for more complex, more luminous systems.

More ultra-faint galaxies need to be extensively studied with photometry and spectroscopy from a chemical and kinematic point of view to assess the nature of as many as possible of the faint dwarfs currently known. Only then can be better understand the early evolution of galaxies and the details of the associated star formation, chemical enrichment and overall growth mechanisms and timescales of these ancient systems.

A.F. is supported by NSF CAREER grant AST-1255160. She also acknowledges support from the Silverman (1968) Family Career Development Professorship. Studies at RSAA, ANU, of the Galaxy's most metal-poor stars and ultra-faint satellite systems are supported by Australian Research Council grants DP0663562, DP0984924, DP120100475, and DP150100862 which J.E.N. gratefully acknowledges. This work made use of NASA's Astrophysics Data System Bibliographic Services.

Facility: Magellan-Clay (MIKE).

\section{REFERENCES}

Adén, D., Eriksson, K., Feltzing, S., et al. 2011, A\&A, 525, 153 Alonso, A., Arribas, S., \& Martínez-Roger, C. 1999, A\&AS, 140, 261 Aoki, W., Beers, T. C., Christlieb, N., et al. 2007, ApJ, 655, 492 Aoki, W., Honda, S., Beers, T. C., et al. 2005, ApJ, 632, 611 Asplund, M., Grevesse, N., Sauval, A. J., \& Scott, P. 2009, ARA\&A, 47, 481 Barklem, P. S., Christlieb, N., Beers, T. C., et al. 2005, A\&A, 439, 129 Beers, T. C., \& Christlieb, N. 2005, ARA\&A, 43, 531

Belokurov, V., Zucker, D. B., Evans, N. W., et al. 2006, ApJL, 647, L111 Bernstein, R., Shectman, S. A., Gunnels, S. M., Mochnacki, S., \& Athey, A. E. 2003, Proc. SPIE, 4841, 1694

Bland-Hawthorn, J., Krumholz, M. R., \& Freeman, K. 2010, ApJ, 713, 166 Brown, T. M., Tumlinson, J., Geha, M., et al. 2014, MmSAI, 85, 493 Casey, A. R. 2014, arXiv:1405.5968

Castelli, F., \& Kurucz, R. L. 2004, arXiv:astro-ph/0405087

Cayrel, R., Depagne, E., Spite, M., et al. 2004, A\&A, 416, 1117

Chiappini, C., Frischknecht, U., Meynet, G., et al. 2011, Natur, 472, 454

Cooke, R. J., \& Madau, P. 2014, ApJ, 791, 116

Feltzing, S., Eriksson, K., Kleyna, J., \& Wilkinson, M. I. 2009, A\&A, 508, L1 François, P., Depagne, E., Hill, V., et al. 2007, A\&A, 476, 935

Frebel, A. 2010, AN, 331, 474

Frebel, A., \& Bromm, V. 2012, ApJ, 759, 115

Frebel, A., Casey, A. R., Jacobson, H. R., \& Yu, Q. 2013, ApJ, 769, 57

Frebel, A., Johnson, J. L., \& Bromm, V. 2007, MNRAS, 380, L40

Frebel, A., \& Norris, J. E. 2015, ARA\&A, 53, 631

Frebel, A., Simon, J. D., Geha, M., \& Willman, B. 2010, ApJ, 708, 560

Frebel, A., Simon, J. D., \& Kirby, E. N. 2014, ApJ, 786, 74

Fulbright, J. P. 2000, AJ, 120, 1841

Fulbright, J. P., Rich, R. M., \& Castro, S. 2004, ApJ, 612, 447

Gilmore, G., Norris, J. E., Monaco, L., et al. 2013, ApJ, 763, 61

Greif, T. H., Springel, V., White, S. D. M., et al. 2011, ApJ, 737, 75

Hollek, J. K., Frebel, A., Roederer, I. U., et al. 2011, ApJ, 742, 54

Hughes, J., Wallerstein, G., \& Bossi, A. 2008, AJ, 136, 2321

Hughes, J., Wallerstein, G., Dotter, A., \& Geisler, D. 2014, MNRAS, 439, 788 Ishigaki, M. N., Aoki, W., Arimoto, N., \& Okamoto, S. 2014, A\&A, 562, A146

Ji, A. P., Frebel, A., \& Bromm, V. 2015, MNRAS, 454, 659

Ji, A. P., Frebel, A., Chiti, A., \& Simon, J. D. 2016a, Natur, 531, 610

Ji, A. P., Frebel, A., Simon, J. D., \& Geha, M. 2016b, ApJ, 817, 41

Jordi, K., Grebel, E. K., \& Amman, K. 2006, A\&A, 460, 339

Karlsson, T., Bland-Hawthorn, J., Freeman, K. C., \& Silk, J. 2012, ApJ, 759,111

Kelson, D. D. 2003, PASP, 115, 688 
Kim, Y.-C., Demarque, P., Yi, S. K., \& Alexander, D. R. 2002, ApJS, 143, 499 Kirby, E. N., Lanfranchi, G. A., Simon, J. D., Cohen, J. G., \& Guhathakurta, P. 2011, ApJ, 727, 78

Kirby, E. N., Simon, J. D., Geha, M., Guhathakurta, P., \& Frebel, A. 2008, ApJL, 685, L43

Koch, A., Feltzing, S., Adén, D., \& Matteucci, F. 2013, A\&A, 554, A5

Koch, A., McWilliam, A., Grebel, E. K., Zucker, D. B., \& Belokurov, V. 2008, ApJL, 688, L13

Koch, A., \& Rich, R. M. 2014, ApJ, 794, 89

Koposov, S. E., Gilmore, G., Walker, M. G., et al. 2011, ApJ, 736, 146

Lai, D. K., Lee, Y. S., Bolte, M., et al. 2011, ApJ, 738, 51

Martin, N. F., Ibata, R. A., Chapman, S. C., Irwin, M., \& Lewis, G. F. 2007, MNRAS, 380, 281

Meynet, G., Ekström, S., \& Maeder, A. 2006, A\&A, 447, 623

Muñoz, R. R., Carlin, J. L., \& Frinchaboy, P. M. a. 2006, ApJL, 650, L51

Norris, J. E., Gilmore, G., Wyse, R. F. G., et al. 2008, ApJL, 689, L113

Norris, J. E., Gilmore, G., Wyse, R. F. G., Yong, D., \& Frebel, A. 2010a, ApJL, 722, L104

Norris, J. E., Wyse, R. F. G., Gilmore, G., et al. 2010b, ApJ, 723, 1632
Norris, J. E., Yong, D., Bessell, M. S., et al. 2013, ApJ, 762, 28

Norris, J. E., Yong, D., Gilmore, G., \& Wyse, R. F. G. 2010c, ApJ, 711,350

Okamoto, S., Arimoto, N., Yamada, Y., \& Onodera, M. 2012, ApJ, 744, 96

Placco, V. M., Frebel, A., Beers, T. C., \& Stancliffe, R. J. 2014, ApJ, 797, 21

Romano, D., Bellazzini, M., Starkenburg, E., \& Leaman, R. 2015, MNRAS, 446, 4220

Siegel, M. H. 2006, ApJL, 649, L83

Simon, J. D., Frebel, A., McWilliam, A., Kirby, E. N., \& Thompson, I. B. 2010, ApJ, 716, 446

Simon, J. D., Geha, M., Minor, Q. E., et al. 2011, ApJ, 733, 46

Smith, B. D., Wise, J. H., O'Shea, B. W., Norman, M. L., \& Khochfar, S. 2015, MNRAS, 452, 2822

Sneden, C. A. 1973, PhD thesis, The Univ. Texas

Sobeck, J. S., Kraft, R. P., Sneden, C., et al. 2011, AJ, 141, 175

Umeda, H., \& Nomoto, K. 2003, Natur, 422, 871

Venn, K. A., Irwin, M., Shetrone, M. D., et al. 2004, AJ, 128, 1177

Webster, D., Frebel, A., \& Bland-Hawthorn, J. 2016, ApJ, 818, 80

Yong, D., Norris, J. E., Bessell, M. S., et al. 2013, ApJ, 762, 26 